\title{
A Fault Analysis Method for Three-Phase Induction Motors Based on Spiking Neural P Systems
}

\author{
Zhu Huang $\left(\mathbb{D},{ }^{1}\right.$ Tao Wang $\mathbb{D},{ }^{1,2}$ Wei Liu $\mathbb{D}^{1},{ }^{1}$ Luis Valencia-Cabrera $\mathbb{D}^{\mathrm{D}}{ }^{3}$ \\ Mario J. Pérez-Jiménez $\mathbb{D}^{3}{ }^{3}$ and Pengpeng $L i \mathbb{D}^{4}$ \\ ${ }^{1}$ School of Electrical Engineering and Electronic Information, Xihua University, Chengdu 610039, China \\ ${ }^{2}$ Key Laboratory of Fluid and Power Machinery, Ministry of Education, Xihua University, Chengdu 610039, China \\ ${ }^{3}$ Research Group on Natural Computing, Department of Computer Science and Artificial Intelligence, Universidad de Sevilla, \\ Sevilla 41012, Spain \\ ${ }^{4}$ Taizhou Power Supply Company, State Grid Zhejiang Electric Power Co. Ltd., Taizhou 318000, China
}

Correspondence should be addressed to Tao Wang; wangatao2005@163.com

Received 19 June 2020; Revised 1 December 2020; Accepted 28 December 2020; Published 13 January 2021

Academic Editor: Zong Woo Geem

Copyright @ 2021 Zhu Huang et al. This is an open access article distributed under the Creative Commons Attribution License, which permits unrestricted use, distribution, and reproduction in any medium, provided the original work is properly cited.

\begin{abstract}
The fault prediction and abductive fault diagnosis of three-phase induction motors are of great importance for improving their working safety, reliability, and economy; however, it is difficult to succeed in solving these issues. This paper proposes a fault analysis method of motors based on modified fuzzy reasoning spiking neural P systems with real numbers (rMFRSNPSs) for fault prediction and abductive fault diagnosis. To achieve this goal, fault fuzzy production rules of three-phase induction motors are first proposed. Then, the rMFRSNPS is presented to model the rules, which provides an intuitive way for modelling the motors. Moreover, to realize the parallel data computing and information reasoning in the fault prediction and diagnosis process, three reasoning algorithms for the rMFRSNPS are proposed: the pulse value reasoning algorithm, the forward fault prediction reasoning algorithm, and the backward abductive fault diagnosis reasoning algorithm. Finally, some case studies are given, in order to verify the feasibility and effectiveness of the proposed method.
\end{abstract}

\section{Introduction}

As an important part of industrial and agricultural productions, the normal operation of three-phase induction motors plays a pivotal role in economic benefits and security risks. For a motor, any potential failure that cannot be predicted or detected in time may produce damage on it, resulting in downtime with potentially huge economic losses [1-4]. In addition, when a motor has faults and is shut down, the first task is to perform abductive fault diagnosis to find its failure causes, which can effectively help the operation and maintenance personnels to locate faulty parts quickly. Therefore, fault prediction and abductive fault diagnosis are of great significance for improving the working reliability and stability of motors [5].

The fault prediction of a motor is usually realized based on an online monitoring system to detect the early failure symptoms and trend parameters that can reflect hidden troubles. Then, these symptoms and parameters are processed by prediction algorithms to obtain early-warning information and integrated decision making [6] to prevent motor failures. For example, [7] diagnosed mechanical faults of motors by vibration analysis, which was carried out through a noncontact approach based on an optical computer mouse and a digital signal processing device. Reference [8] proposed a two-stage machine learning analysis architecture, where a recurrent neural network-based variational autoencoder was proposed in the first stage, and principal components analysis and linear discriminant analysis techniques were applied in the second stage. This architecture was useful to accurately predict the motor fault modes only by using motor vibration time-domain signals. In [9], a hybrid technique for bearing prognostics was proposed, which utilized a regression-based adaptive prediction model to find the evolution trend of bearing health indices. However, so far, most fault prediction methods require a huge number of historical data to perform the training and learning processes of their predictive models. 
The abductive fault diagnosis of a motor consists in finding failure causes from its fault phenomena and operation data, so that a motor can be effectively repaired, thus reducing economic losses [10]. In [11], an instantaneous frequency analysis method based on abnormal sounds was proposed. However, when the acoustic signals of a motor were mixed by other acoustic signals (such as reflected signals and overlapped signals), it was difficult to extract the features of bearing fault information. In [12], a new current signature analysis-based fault detector for motors based on a matched subspace technique was proposed. However, it was only effective for detecting eccentricity faults, bearing faults, and broken rotor bars. Reference [13] proposed a technique based on vibration information to identify and classify different bearing failure conditions. The setting and testing of parameters was strict and difficult; for example, the accelerometer needs to be very close to the motor, and the setting of accelerometer and data logger should be the same. However, this method needed much historical data with a complex computing process. In [14], an intelligent fault diagnosis of three-phase induction motors using a signal-based method was proposed and tested in different situations, in order to verify its availability in diagnosing failures, even when the operating mode data were limited. However, the experimental results showed that it was only suitable for the diagnosis of broken bars and bearing failure.

The aforementioned methods have their own advantages with the same disadvantages implying that they mainly focus on the diagnosis of a single fault, such as the rotor bar breaking or the stator short circuit. Thus, they cannot effectively diagnose multiple faults, not achieving the requirement of performing an overall fault analysis of the whole machine.

Therefore, how to improve the abovementioned fault prediction and abductive fault diagnosis methods or put forward new ones is the main issue in the corresponding engineering domain for the motors. On the other hand, with the rapid development of artificial intelligence technology, intelligent analysis and diagnosis methods are gradually developed, such as expert systems (ESs) [15], artificial neural networks (ANNs) [16-20], Petri nets (PNs) [21-23], tissue P systems (TPSs) [24-26], and spiking neural P systems (SNPSs) [27-34]. Specifically, SNPS is a novel high-performance bioinspired distributed parallel computing model with powerful information processing ability. It is a special kind of neural-like P system [29] inspired by the topological structure of biological neural networks and the way that biological neurons store, transmit, and exchange messages, i.e., by sending electrical impulses (spikes) along axons in a distributed and parallel manner [30-32].

The SNPS-based fault diagnosis methods (for example, the ones for power systems) are derived from the similarities between the pulse transmission between neurons via synapses and the fault propagation in power systems. Accordingly, the basic mechanism to address fault diagnosis based on SNPSs is to find faulty sections by dealing with the uncertainty [35] of fault alarm information. In general, the input neurons of an SNPS correspond to protective devices (including protective relays and circuit breakers), and the output neurons are associated with suspicious fault sections. Thus, the pulse values of input neurons represent the action information of protective devices, that is, the actual tripping information from the supervisory control and data acquisition system or the action confidence levels represented by fuzzy numbers [36]. On the other hand, the pulse values of output neurons express the trip information $o$ fault confidence levels of the suspicious sections. When the fault reasoning is finished, faulty sections are finally determined based on the fault confidence levels according to criterion rules.

Because of the high requirement of fault diagnosis methods for processing fault information, the SNPS-based diagnosis methods have become a hot research topic with rich research results $[27-29,33,34]$. However, up to now, the relevant research work is mainly focused on the fault diagnosis of power systems. Besides, the existing work mainly studies the postevent diagnosis problems. Therefore, to give full play to the excellent information processing ability and computing power of SNPSs, it is of great importance to expand their scope to different application fields, as well as extend the applications from the postante ones to new ex-ante analysis and prediction frameworks.

Therefore, this paper moves forward in this widening of the scope of SNPSs. More specifically, the work proposes a fault analysis method based on modified fuzzy reasoning spiking neural P systems with real numbers (rMFRSNPSs) for threephase induction motors. As an important part of this new method presented here, the forward fault prediction reasoning algorithm (FFPRA) and the backward abductive fault diagnosis reasoning algorithm (BAFDRA) are proposed. The main contributions of this paper are described as follows:

(1) Based on the existing variants of SNPSs, we propose a modified fuzzy reasoning spiking neural $\mathrm{P}$ system with real numbers by simplifying previously existing ones. In order to enable the rMFRSNPSs to achieve fault prediction and abductive diagnosis, three algorithms are proposed, i.e., the pulse value reasoning algorithm (PVRA), the FFPRA, and the BAFDRA, respectively.

(2) Fault fuzzy production rules for motors are presented to obtain the relationships between failure symptoms and different faults. Moreover, the rMFRSNPS-based model for a motor is built via modelling the production rules, which is the basis for the fault analysis from the point of view of a whole machine.

(3) Firstly, the SNPS is introduced to solve the fault diagnosis of motors, including forward fault prediction and backward abductive fault diagnosis. In addition, we also extend its application from the postante diagnosis to a new ex-ante prediction framework. The new framework not only can take full advantages of the SNPS for the fault prediction with potential fault paths and their occurrence probabilities in an ex-ante prediction problem but also can effectively find failure causes with abductive reasoning paths and their probabilities in a postante fault diagnosis problem.

\section{The rMFRSNPS-Based Fault Model for Motors}

In this section, we first present the rMFRSNPS and then propose fault fuzzy production rules of motors. Finally, the 
rMFRSNPS is employed to model the rules to propose a universal rMFRSNPS-based fault analysis model.

\subsection{Modified Fuzzy Reasoning Spiking Neural P Systems with Real Numbers}

Definition 1. A modified fuzzy reasoning spiking neural $\mathrm{P}$ system with real numbers (rMFRSNPS, for short) of degree $m \geq 1$ is a tuple

$$
\prod=\left(O, \sigma_{1}, \ldots, \sigma_{m}, \text { syn, in, out }\right),
$$

where

(1) $O=\{a\}$ is a singleton alphabet ( $a$ is called a spike, $O$ is a set of spikes).

(2) $Q=Q_{p} \cup Q_{r}$ is a neuron set, where $Q_{p}=\left\{\sigma_{1}, \ldots, \sigma_{s}\right\}$ is the proposition neuron set and $Q_{r}=\left\{\sigma_{s+1}, \ldots, \sigma_{s+t}\right\}$ is the rule neuron set, being $s+t=m$. Each proposition neuron $\sigma_{i}$ is of the form $\left(\alpha_{i}, \lambda_{i}, r_{i}\right), 1 \leq i \leq s$, where

(a) $\alpha_{i}$ is a real number in $[0,1]$ representing the potential value of spikes (i.e., value of electrical impulses) contained in $\sigma_{i}$.

(b) $\lambda_{i}$ is a real number in $(0,1)$ representing the firing threshold of $\sigma_{i}$.

(c) $r_{i}$ represents a firing (spiking) rule of proposition neuron $\sigma_{i}$ with the form $E / a^{\alpha} \longrightarrow a^{\theta}$, where $\alpha$ and $\theta$ are real numbers in [0,1], and $E=\left\{a^{n} \wedge \alpha \geq \lambda_{i}\right\}$ is the firing condition. The firing rule $r_{i}$ of $\sigma_{i}$ can be applied if and only if $\sigma_{i}$ receives, at least, $n$ spikes and the potential value of spikes satisfies that $\alpha \geq \lambda_{i}$. By applying rule $r_{i}, \sigma_{i}$ will consume (remove) a spike with pulse value $\alpha$ and then not only produce (emit) a new spike with pulse value $\theta$ but also transmit it to its postsynaptic neurons.

Each rule neuron $\sigma_{s+j}$ is of the form $\left(\delta_{j}, c_{j}, \lambda_{j}, r_{j}\right), 1 \leq j \leq t$, where

(a) $\delta_{j}$ is a real number in $[0,1]$ representing the potential value of spikes (i.e., value of electrical impulses) contained in $\sigma_{s+j}$.

(b) $c_{j}$ is a real number in $[0,1]$ representing the truth value of $\sigma_{s+j}$.

(c) $\lambda_{j}$ is a real number in $(0,1)$ representing the firing threshold of $\sigma_{s+j}$.

(d) $r_{j}$ represents a firing (spiking) rule of $\sigma_{s+j}$ with the form $E / a^{\delta} \longrightarrow a^{\beta}$, where $\delta$ and $\beta$ are real numbers in $[0,1]$, and $E=\left\{a^{n} \wedge \delta \geq \lambda_{j}\right\}$ is the firing condition. The firing rule $r_{j}$ of $\sigma_{s+j}$ can be applied if and only if $\sigma_{s+j}$ receives, at least, $n$ spikes and the potential value of spikes satisfies that $\delta \geq \lambda_{j}$. By applying rule $r_{j}, \sigma_{s+j}$ will consume (remove) a spike with pulse value $\delta$ and then not only produce (emit) a new spike with pulse value $\beta$ but also transmit it to its postsynaptic neurons.

(3) $\operatorname{syn} \subseteq\{1, \ldots, m\} \times\{1, \ldots, m\}$ with $\quad(i, i) \notin$ syn for $1 \leq i \leq m$, is a directed graph of synapses between linked neurons.
(4) in $\subseteq\{1, \ldots, m\}$ and out $\subseteq\{1, \ldots, m\}$ represent the sets of input and output neurons of $\prod$, respectively.

Fuzzy production rules can be modelled in the framework of rMFRSNPSs. Let us recall that there are, basically, three types of fuzzy production rules [33].

(a) GENERAL rule, whose format is

$R_{i}\left(\mathrm{CF}=c_{i}\right): \operatorname{IF} p_{1}\left(\alpha_{1}\right)$ THEN $p_{2}\left(\alpha_{2}\right)$, where $p_{1}$ is an antecedent proposition and $p_{2}$ is a consequent proposition

(b) Compound AND rule, whose format is

$R_{i}\left(\mathrm{CF}=c_{i}\right):$ IF $p_{1}\left(\alpha_{1}\right)$ AND ...AND $p_{k-1}\left(\alpha_{k-1}\right)$ THEN $p_{k}\left(\alpha_{k}\right)$, where $p_{1}, \ldots, p_{k-1}$ are antecedent propositions, $p_{k}$ is a consequent proposition, and $k \geq 3$

(c) Compound OR rule, whose format is $R_{i}\left(\mathrm{CF}=c_{i}\right):$ IF $p_{1}\left(\alpha_{1}\right)$ OR...OR $p_{k-1}\left(\alpha_{k-1}\right)$ THEN $p_{k}\left(\alpha_{k}\right)$, where $p_{1}, \ldots, p_{k-1}$ are antecedent propositions, $p_{k}$ is a consequent proposition, and $k \geq 3$

In fact, there exists another type of rule whose format is $R_{i}\left(\mathrm{CF}=c_{i}\right):$ IF $p_{1}\left(\alpha_{1}\right)$ THEN $p_{2}\left(\alpha_{2}\right)$ AND $\ldots$ AND $p_{k}\left(\alpha_{k}\right)$, where $p_{1}$ is an antecedent proposition and $p_{2}, \ldots, p_{k}$ are consequent propositions, with $k \geq 3$. However, this kind of rules can be considered as a particular case of a composition of $k-1$ GENERAL rules.

In order to model fuzzy production rules by means of rMFRSNPSs, a proposition neuron in an rMFRSNPS is associated with a proposition in the fuzzy production rules. Such neurons will be represented by a circle. If a proposition neuron $\sigma_{i}=\left(\alpha_{i}, \lambda_{i}, r_{i}\right)$ is an input neuron, then its initial potential value $\alpha_{i}$ represents the information that $\sigma_{i}$ has received from the environment.

A general rule neuron in an rMFRSNPS consists of only one presynaptic proposition neuron and one or more postsynaptic proposition neurons. Therefore, in a natural manner, a general rule neuron can be associated with a general rule, that is, with a fuzzy production rule which has only one proposition on its antecedent part. An and rule neuron in an rMFRSNPS consists of, at least, two presynaptic proposition neurons with an AND relationship among them and only one postsynaptic proposition neuron. Thus, in a straightforward way, an and rule neuron can be associated with each compound AND fuzzy production rule. Finally, an or rule neuron in an rMFRSNPS consists of, at least, two presynaptic proposition neurons with an OR relationship among them and only one postsynaptic proposition neuron. According to the previous comments, an or rule neuron can be associated with each compound OR fuzzy production rule. These rule neurons are represented by a rectangle, and they are graphically illustrated in Figure 1.

2.2. Fault Fuzzy Production Rules for Motors. In this paper, the possible failures in a motor include electrical faults and mechanical ones. The first class includes failures such as the excessive current in a phase, the excessive excitation current, a phase voltage loss, the phase-absent operation, the three-phase 
current asymmetry, and the insulation winding burned down. The second class contains failures such as the bearing expansion by heat, the excessive wear of bearing, the excessive vibration of motor in operation, the abnormal noise, the rotor stuck or stopped rotating, and the motor sweeping. According to the principle of motor failures [23, 37-41] and the fault simulation model in Figure 2, fault fuzzy production rules of motors are obtained as follows, where events corresponding to the propositions in the rules are shown in Table 1.

Rule $1\left(c_{1}\right)$ : IF $p_{1}$ OR $p_{2}$ occurs, THEN $p_{17}$ occurs

Rule $2\left(c_{2}\right)$ : IF $p_{2}$ AND $p_{3}$ occur, THEN $p_{18}$ occurs

Rule $3\left(c_{3}\right)$ : IF $p_{3}$ occurs, THEN $p_{19}$ occurs

Rule $4\left(c_{4}\right)$ : IF $p_{4}$ occurs, THEN $p_{20}$ occurs

Rule $5\left(c_{5}\right)$ : IF $p_{5}$ occurs, THEN $p_{21}$ occurs

Rule $6\left(c_{6}\right)$ : IF $p_{6}$ OR $p_{7}$ occurs, THEN $p_{22}$ occurs

Rule $7\left(c_{7}\right)$ : IF p8 occurs, THEN $p_{23}$ occurs

Rule $8\left(c_{8}\right)$ : IF $p_{8}$ occurs, THEN $p_{24}$ occurs

Rule $9\left(c_{9}\right)$ : IF $p_{9}$ OR $p_{10}$ occurs, THEN $p_{25}$ occurs

Rule $10\left(c_{10}\right)$ : IF $p_{10}$ OR $p_{11}$ occurs, THEN $p_{26}$ occurs

Rule $11\left(c_{11}\right)$ : IF $p_{12}$ OR $p_{13}$ occurs, THEN $p_{27}$ occurs

Rule $12\left(c_{12}\right)$ : IF $p_{14}$ occurs, THEN $p_{28}$ occurs

Rule 13 ( $\left.c_{13}\right)$ : IF $p_{15}$ AND $p_{16}$ occur, THEN $p_{29}$ occurs

Rule $14\left(c_{14}\right)$ : IF $p_{17}$ OR $p_{18}$ OR $p_{19}$ occurs, THEN $p_{30}$ occurs

Rule $15\left(c_{15}\right)$ : IF $p_{20}$ occurs, THEN $p_{31}$ occurs

Rule $16\left(c_{16}\right)$ : IF $p_{21}$ occurs, THEN $p_{32}$ occurs

Rule $17\left(c_{17}\right)$ : IF $p_{22}$ occurs, THEN $p_{33}$ occurs

Rule $18\left(c_{18}\right)$ : IF $p_{22}$ OR $p_{23}$ occurs, THEN $p_{34}$ occurs

Rule $19\left(c_{19}\right)$ : IF $p_{24}$ occurs, THEN $p_{35}$ occurs

Rule $20\left(c_{20}\right)$ : IF $p_{25}$ occurs, THEN $p_{36}$ occurs

Rule $21\left(c_{21}\right)$ : IF $p_{26}$ occurs, THEN $p_{37}$ occurs

Rule $22\left(c_{22}\right)$ : IF $p_{27}$ occurs, THEN $p_{38}$ occurs

Rule $23\left(c_{23}\right)$ : IF $p_{28}$ occurs, THEN $p_{39}$ occurs

Rule $24\left(c_{24}\right)$ : IF $p_{29}$ occurs, THEN $p_{40}$ occurs

Rule $25\left(c_{25}\right)$ : IF $p_{30}$ OR $p_{31}$ OR $p_{32}$ OR $\sigma_{33}$ occurs, THEN $p_{41}$ occurs

Rule $26\left(c_{26}\right)$ : IF $p_{34}$ OR $p_{35}$ occurs, THEN $p_{42}$ occurs Rule $27\left(c_{27}\right)$ : IF $p_{36}$ occurs, THEN $p_{43}$ occurs

Rule $28\left(c_{28}\right)$ : IF $p_{37}$ OR $p_{38}$ occurs, THEN $p_{44}$ occurs

Rule $29\left(c_{29}\right)$ : IF $p_{39}$ OR $p_{40}$ occurs, THEN $p_{45}$ occurs

Rule $30\left(c_{30}\right)$ : IF $p_{41}$ occurs, THEN $p_{46}$ occurs

Rule $31\left(c_{31}\right)$ : IF $p_{42}$ OR $p_{43}$ occurs, THEN $p_{47}$ occurs

Rule $32\left(c_{32}\right)$ : IF $p_{44}$ occurs, THEN $p_{48}$ occurs

Rule $33\left(c_{33}\right)$ : IF $p_{45}$ occurs, THEN $p_{49}$ occurs

Rule $34\left(c_{34}\right)$ : IF $p_{46}$ OR $p_{47}$ OR $p_{48}$ OR $p_{49}$ occurs, THEN $p_{50}$ occurs
2.3. The rMFRSNPS-Based Model for a Motor. This section models the fault fuzzy production rules proposed in Section 2.2 and builds a universal rMFRSNPS-based fault analysis model for three-phase induction motors, as shown in Figure 3. The designed rMFRSNPS is of degree $m=84$ and specifically contains $s=50$ proposition neurons and $t=34$ rule neurons.

\section{Fault Analysis Method Based on rMFRSNPSs}

This section proposes a fault analysis method based on rMFRSNPSs for three-phase induction motors, whose flowchart is shown in Figure 4 , where $\mathbf{0}=(0, \ldots, 0)_{t \times 1}^{T}$. The proposed method includes two parts, one is for fault prediction before fault occurrence while the other one is for abductive diagnosis reasoning after failures. Moreover, a diagrammatic sketch of the application scenario for the proposed method is shown in Figure 5, where red circles represent the already happened events while blue circles express the not occurred ones. The status of a motor is monitored in real time. When the motor has fault symptoms or faults, relevant state data will be transmitted to the fault analysis center, where our method will be used to handle the events.

Specifically, in this proposed method, the PVRA (Algorithm 1) is proposed to get the potential value of spikes in neurons using historical data and expertise. When a motor has no faults, but is accompanied by fault symptoms, the FFPRA (Algorithm 2) is employed to predict propagation paths with occurrence probabilities. When a motor fails, the fault positions (corresponding to neurons with fault pulses) are found according to failure phenomena, and then, failure causes with probabilities are obtained according to the BAFDRA (Algorithm 3). Thus, the maintenance efficiency can be improved accordingly to check the motor on the basis of results got by the prediction reasoning or abductive reasoning. Note that the historical data include fault probabilities of fault events (Algorithms 1-3), certainty factors of fault production rules (Algorithms 1 and 2), and the tightness degree between related fault events (Algorithm 3).

Next, we describe Algorithms 1-3 in detail as follows.

3.1. Pulse Value Reasoning Algorithm. To explain this algorithm, we introduce its vectors, matrices, and operators as follows (PN denotes proposition neuron and RN denotes rule neuron):

(1) $\boldsymbol{\alpha}=\left(\alpha_{1}, \ldots, \alpha_{s}\right)^{T}$ is a pulse value vector of PNs, where $\alpha_{i}(i=1, \ldots, s)$ represents the pulse value of the $i$-th PN $\sigma_{i}$. If a PN has not any pulse, then its pulse value is 0 .

(2) $\boldsymbol{\delta}=\left(\delta_{1}, \ldots, \delta_{t}\right)^{T}$ is a pulse value vector of RNs, where $\delta_{j}(j=1, \ldots, t)$ is the pulse value of the $j$-th $\mathrm{RN} \sigma_{s+j}$. If an RN has not any pulse, then its pulse value is 0 . 

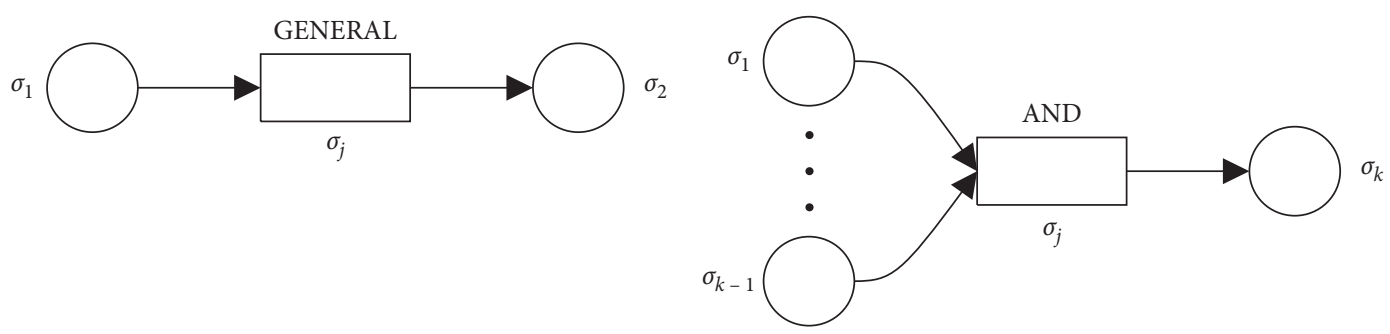

(a)

(b)

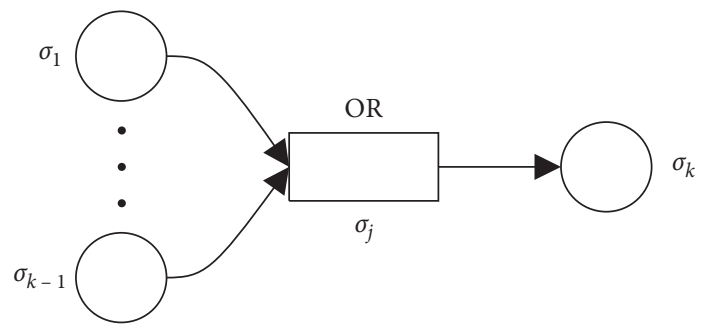

(c)

FIGURE 1: rMFRSNPS-based models for fuzzy fault production rules. (a) GENERAL rule; (b) compound AND rule; and (c) compound OR rule.

(3) $\lambda_{p}=\left(\lambda_{p_{1}}, \ldots, \lambda_{p_{s}}\right)^{T}$ is a firing threshold vector of PNs.

(4) $\boldsymbol{\lambda}_{r}=\left(\lambda_{r_{1}}, \ldots, \lambda_{r_{t}}\right)^{T}$ is a firing threshold vector of RNs.

(5) $\mathbf{C}=\operatorname{diag}\left(c_{1}, \ldots, c_{t}\right)$ is a diagonal matrix of truth values of RNs, where $c_{j}(j=1, \ldots, t)$ is the truth value of the $j$-th $\mathrm{RN} \sigma_{s+j}$.

(6) $\mathbf{D}_{1}=\left(d_{i j}\right)_{s \times t}$ is a synaptic matrix, which represents the directed synaptic connections from PNs to general RNs. If there is a synapse from the $\mathrm{PN} \sigma_{i}$ to the general $\mathrm{RN} \sigma_{s+j}$, then $d_{i j}=1$; otherwise, $d_{i j}=0$.

(7) $\mathbf{D}_{2}=\left(d_{i j}\right)_{s \times t}$ is a synaptic matrix, which represents the directed synaptic connections from PNs to and RNs. If there is a synapse from the PN $\sigma_{i}$ to the and RN $\sigma_{s+j}$, then $d_{i j}=1$; otherwise, $d_{i j}=0$.

(8) $\mathbf{D}_{3}=\left(d_{i j}\right)_{s \times t}$ is a synaptic matrix, which represents the directed synaptic connections from PNs to or RNs. If there is a synapse from the PN $\sigma_{i}$ to the or RN $\sigma_{s+j}$, then $d_{i j}=1$; otherwise, $d_{i j}=0$.

(9) $\mathbf{D}_{4}=\left(d_{j i}\right)_{t \times s}$ is a synaptic matrix, which represents the directed synaptic connections from RNs to PNs. If there is a synapse from the $\mathrm{RN} \sigma_{s+j}$ to the $\mathrm{PN} \sigma_{i}$, then $d_{j i}=1$; otherwise, $d_{j i}=0$.

(10) $\mathbf{0}=(0, \ldots, 0)_{t \times 1}^{T}$ is null vector.

(11) $\mathbf{D}^{T} * \boldsymbol{\alpha}=\left(\bar{d}_{1}, \ldots, \bar{d}_{t}\right)^{T}$, where $\bar{d}_{j}=d_{1 j} \times \alpha_{1}+\cdots+$ $d_{s j} \times \boldsymbol{\alpha}_{s}, 1 \leq j \leq t$.

(12) $\mathbf{D}^{T} \cdot \boldsymbol{\alpha}=\left(\bar{d}_{1}, \ldots, \bar{d}_{t}\right)^{T}$, where $\bar{d}_{j}=\min \left\{d_{1 j} \times \alpha_{1}+\right.$ $\left.\cdots+d_{s j} \times \alpha_{s}\right\}, 1 \leq j \leq t$.
(13) $\mathbf{D}^{T} \circ \alpha=\left(\bar{d}_{1}, \ldots, \bar{d}_{t}\right)^{T}$, where $\bar{d}_{j}=\max \left\{d_{1 j} \times \alpha_{1}+\right.$ $\left.\cdots+d_{s j} \times \alpha_{s}\right\}, 1 \leq j \leq t$.

3.2. Forward Fault Prediction Reasoning Algorithm. To explain this algorithm, we introduce its vectors, matrices, and operators as follows:

(1) $\mathbf{N}_{p}^{+}$is the number vector of PNs where pulses are located. If a PN contains a pulse, then the number of the neuron in which the pulse occurs is numbered as 1 ; otherwise, it is 0 .

(2) $\mathbf{N}_{r}^{+}$is the number vector of RNs where pulses are located. If an $\mathrm{RN}$ contains a pulse, the number of the neuron in which the pulse occurs is numbered as 1 ; otherwise, it is 0 .

(3) $\mathbf{A} \Delta \mathbf{B}=\left(c_{i 1}\right)_{x \times 1}$, where $\mathbf{A}=\left(a_{i k}\right)_{x \times y}, \mathbf{B}=\left(b_{k 1}\right)_{y \times 1}$, and $c_{i 1}=\max \left\{a_{i k} b_{k 1}\right\}, 1 \leq i \leq x, 1 \leq k \leq y$.

(4) $\mathbf{A} \nabla \mathbf{B}=\left(c_{i 1}\right)_{x \times 1}$, where $\mathbf{A}=\left(a_{i k}\right)_{x \times y}, \mathbf{B}=\left(b_{k 1}\right)_{y \times 1}$. If $\sum_{k=1}^{y} a_{i k} b_{k 1}<2$, then $c_{i 1}=0$, otherwise $c_{i 1}=$ $1,1 \leq i \leq x, 1 \leq k \leq y$.

(5) $\mathbf{A} \otimes \mathbf{B}=\left(c_{i j}\right)_{x \times y}$, where $\mathbf{A}=\left(a_{i j}\right)_{x \times y}, \mathbf{B}=\left(b_{i 1}\right)_{x \times 1}$, and $c_{i j}=a_{i j} b_{i 1}, 1 \leq i \leq x, 1 \leq j \leq y$.

(6) $\mathbf{A} \oplus \mathbf{B}=\left(c_{i j}\right)_{x \times y}$, where $\mathbf{A}=\left(a_{i j}\right)_{x \times y}, \mathbf{B}=\left(b_{i j}\right)_{x \times y}$, and $c_{i j}=\max \left\{a_{i j}, b_{i j}\right\}, 1 \leq i \leq x, 1 \leq j \leq y$.

(7) $\mathbf{A} \Theta \mathbf{B}=\left(c_{i j}\right)_{x \times y}$, where $\mathbf{A}=\left(a_{i j}\right)_{x \times y}, \mathbf{B}=\left(b_{i j}\right)_{x \times y}$. If $a_{i j} \geq b_{i j}$, then $\quad c_{i j}=1$, otherwise, $\quad c_{i j}=0$, $1 \leq i \leq x, 1 \leq j \leq y$.

Note that the vectors $\boldsymbol{\alpha}, \boldsymbol{\delta}, \boldsymbol{\lambda}_{p}, \boldsymbol{\lambda}_{r}$, and $\mathbf{0}$, the matrices $\mathbf{D}_{1}, \mathbf{D}_{2}, \mathbf{D}_{3}, \mathbf{D}_{4}$, and $\mathbf{C}$, and the operators $*, \cdot$ and $\circ$ in Algorithm 2 are the same as the ones in Algorithm 1. 


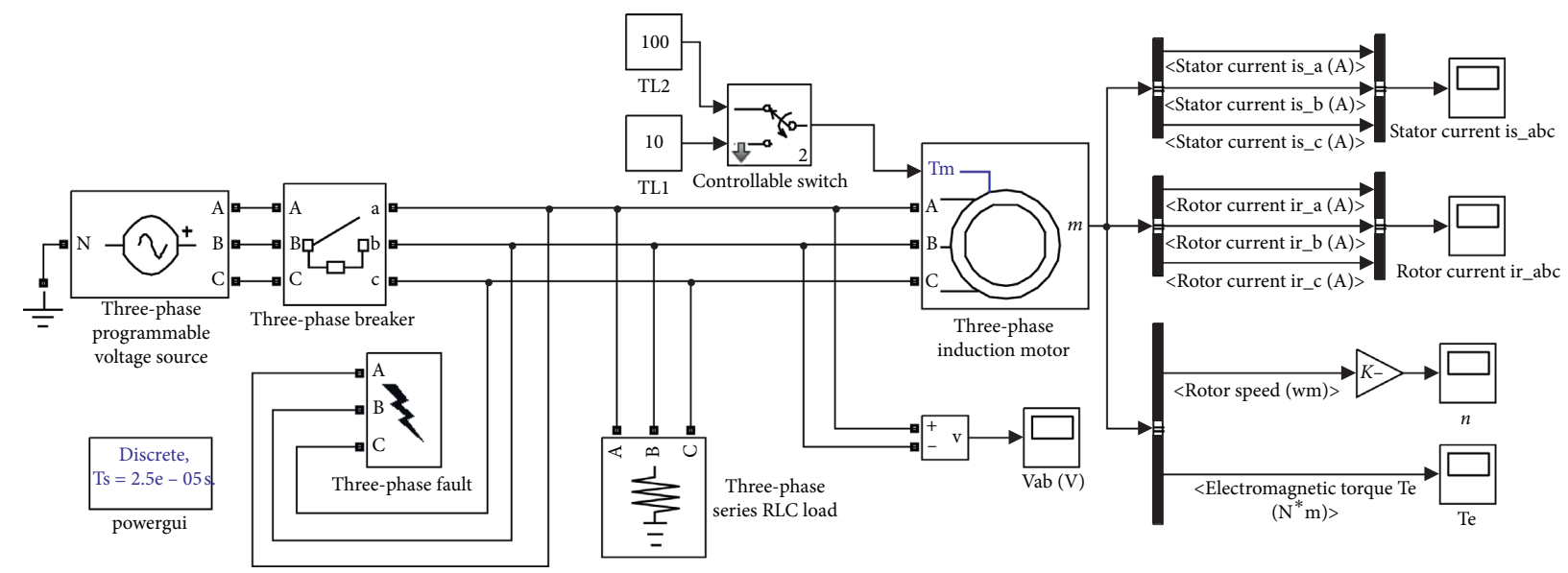

FIgURe 2: Fault simulation model of a three-phase induction motor.

3.3. Backward Abductive Fault Diagnosis Reasoning Algorithm. To improve the accuracy of backward abductive reasoning, this paper integrates a fault screening mechanism of the precise minimum cut set (please see Definition 2) into the parallel reasoning ability of SNPSs to propose the BAFDRA for the rMFRSNPS, i.e., Algorithm 3. The precise minimum cut set effectively combines the abductive principle of top events in minimum cut sets [42] with the screening mechanism, where, in two adjacent fault events, a bottom event corresponds to a fault or a fault symptom and a top event corresponds to a fault. Moreover, the screening mechanism is used to improve the abductive reasoning accuracy by eliminating pulses contained in the minimum cut set whose danger degree is lower than the dangerous threshold, where the danger degree is used to access the fault risk of motors [43].

Definition 2. A precise minimum cut set (PMCS) is a tuple

$$
Q=\left(Q_{1}, \ldots, Q_{l}\right), \quad 1 \leq l \leq s,
$$

where

(1) $Q_{1}, \ldots, Q_{l}$ are $l$ minimum cut sets (MCSs), where

(a) A general rule neuron has one presynaptic proposition neuron (corresponding to a bottom event) and one postsynaptic proposition neuron (corresponding to a top event). For this kind of rule neurons, the top event can be triggered only by the bottom event; thus, the MCS of the top event consists of the presynaptic neuron.

(b) An or rule neuron has more than one presynaptic proposition neurons (each of them corresponds to a bottom event) and only one postsynaptic proposition neuron (corresponding to a top event). For this kind of rule neurons, the top event can be triggered by any bottom event; thus, the MCS of the top event consists of any presynaptic neuron. That is, if there are $l$ bottom events that can trigger the top event, then the top event will have $l$ MCSs with each MCS consisting of one presynaptic neuron corresponding to one of the $l$ bottom events.

(c) An and rule neuron has more than one presynaptic proposition neuron (each of them corresponds to a bottom event) and only one postsynaptic proposition neuron (corresponding to a top event). For this kind of rule neurons, the top event can only be triggered by all bottom events at the same time, so that the MCS of the top event consists of all the presynaptic neurons. That is, if there are $l$ bottom events that can trigger the top event, then the top event will have only one MCS and it should consist of all the presynaptic neurons corresponding to the $l$ bottom events.

(2) $Q_{g}=\left\{y\left(\sigma_{i}\right), y\left(Q_{g}\right), \lambda_{y}\right\}, 1 \leq g \leq l, 1 \leq i \leq s$ is the $g$-th MCS, where

(a) $y\left(\sigma_{i}\right)$ is the danger degree of the $i$-th $\mathrm{PN} \sigma_{i}$, defined as

$$
y\left(\sigma_{i}\right)=w\left(\sigma_{i}\right) \times \alpha_{i},
$$

where $w\left(\sigma_{i}\right)$ is a weighted value in $[0,1]$ representing the tightness degree between $\mathrm{PN} \sigma_{i}$ and its postsynaptic neurons.

(b) $y\left(Q_{g}\right)$ is a danger degree of the $g$-th minimum cut set, i.e., $Q_{g}$, which is defined as

$$
y\left(Q_{g}\right)=\prod_{j=1}^{q} y\left(\sigma_{j}\right), \quad 1 \leq q \leq s .
$$

(c) $\lambda_{y}$ is a number in $(0,1)$ representing the danger degree threshold of an MCS. When the danger 
TABle 1: The meaning of propositions in fuzzy fault production rules.

\begin{tabular}{|c|c|}
\hline Propositions & Events \\
\hline$p_{1}$ & Overload \\
\hline$p_{2}$ & Rotor winding short circuit \\
\hline$p_{3}$ & The resistance value of a phase winding decreases \\
\hline$p_{4}$ & Fuse melt fault \\
\hline$p_{5}$ & Damage of shaft seal ring structure \\
\hline$p_{6}$ & Oil sealing material overheating \\
\hline$p_{7}$ & Excessive roughness value of the seal surface shaft \\
\hline$p_{8}$ & Excessive temperature \\
\hline$p_{9}$ & Mechanical fault of the rotor winding \\
\hline$p_{10}$ & $\begin{array}{l}\text { The motor centerline is inconsistent with the pump } \\
\text { one }\end{array}$ \\
\hline$p_{11}$ & Fault of the bearing locking device \\
\hline$p_{12}$ & Rotor core deformation \\
\hline$p_{13}$ & Fracture or shedding of magnetic slot wedges \\
\hline$p_{14}$ & Dewelding at the joint of the winding and lead wire \\
\hline$p_{15}$ & Connection box joint loosened \\
\hline$p_{16}$ & Poor contact of the power control loop switch \\
\hline$p_{17}$ & Decrease in rotational speed \\
\hline$p_{18}$ & Excessive current in a phase \\
\hline$p_{19}$ & Excessive excitation current \\
\hline$p_{20}$ & A phase voltage loss \\
\hline$p_{21}$ & Foreign matter enters the rotary shaft clearance \\
\hline$p_{22}$ & The motor oil intake \\
\hline$p_{23}$ & $\begin{array}{l}\text { Oxidation and decomposition of bearing } \\
\text { lubricating oil }\end{array}$ \\
\hline$p_{24}$ & Bearing expansion by heat \\
\hline$p_{25}$ & Bearing generates additional load \\
\hline$p_{26}$ & Rotor axial moves \\
\hline$p_{27}$ & The iron core of the stator and rotor has an air gap \\
\hline$p_{28}$ & Rotor winding open circuit \\
\hline$p_{29}$ & Contact resistance value increases \\
\hline$p_{30}$ & Motor overheating \\
\hline$p_{31}$ & Phase-absent operation \\
\hline$p_{32}$ & Abnormal rotation or the rotor is stuck \\
\hline$p_{33}$ & Insulation aging \\
\hline$p_{34}$ & Reduction of lubricant oil \\
\hline$p_{35}$ & $\begin{array}{l}\text { Friction occurs between the crankshaft ring and } \\
\text { shaft hole }\end{array}$ \\
\hline$p_{36}$ & Excessive vibration of the motor in operation \\
\hline$p_{37}$ & Excessive bearing noise \\
\hline$p_{38}$ & Motor sweeping \\
\hline$p_{39}$ & Three-phase current of the stator increases \\
\hline$p_{40}$ & Increased pressure drop \\
\hline$p_{41}$ & Three-phase current asymmetry \\
\hline$p_{42}$ & Excessive wear of the bearing \\
\hline$p_{43}$ & Irregular impact load \\
\hline$p_{44}$ & Abnormal noise \\
\hline$p_{45}$ & $\begin{array}{c}\text { Rotor rotation is weak or the rotor does not turn } \\
\text { and hum }\end{array}$ \\
\hline$p_{46}$ & Insulation winding burned down \\
\hline$p_{47}$ & Motor axle holder \\
\hline$p_{48}$ & Rotor stuck or stopped rotating \\
\hline$P_{49}$ & The motor appears local high heat \\
\hline$p_{50}$ & The motor cannot work \\
\hline - & - \\
\hline
\end{tabular}

degree of an MCS is greater than $\lambda_{y}$, then the MCS is called a PMCS and the PNs with pulse in the PMCS form the fault paths, with the first PN in each path being called the fault source.

Algorithm 3 is shown as follows:

To explain the algorithm, we introduce its vectors, matrices, and operators as follows:

(1) $\mathbf{N}_{p}^{-}$is the number vector of PNs where fault pulses are located. If a PN contains a fault pulse, then the number of the neuron is numbered as 1 ; otherwise, it is 0 .

(2) $\mathbf{N}_{r}^{-}$is the number vector of RNs where fault pulses are located. If a rule neuron contains a fault pulse, then the number of the neuron is numbered as 1; otherwise, it is 0 .

(3) $\boldsymbol{\theta}_{p}=\left(\theta_{p_{1}}, \ldots, \theta_{p_{s}}\right)^{T}$ is a fault pulse value vector of PNs, where $\theta_{p_{i}}(i=1, \ldots, s)$ represents the pulse value of the $i$-th $\mathrm{PN} \sigma_{i}$. If a $\mathrm{PN}$ has not any pulse, then its pulse value is 0 .

(4) $\boldsymbol{\theta}_{r}=\left(\theta_{r}, \ldots, \theta_{r_{t}}\right)^{T}$ is a fault pulse value vector of RNs, where $\theta_{r_{i}}(j=1, \ldots, t)$ represents the pulse value of the $j$-th $\mathrm{RN} \sigma_{s+j}$. If a RN has not any pulse, then its pulse value is 0 .

(5) $\lambda_{y}$ is a dangerous threshold of an MCS.

(6) $\mathbf{W}=\left[\begin{array}{ccccc}w_{11}\left(\sigma_{1}\right) & \ldots & w_{1 k}\left(\sigma_{1}\right) & \ldots & w_{1 s}\left(\sigma_{1}\right) \\ & \ddots & & & \\ \vdots & & w_{i k}\left(\sigma_{i}\right) & & \vdots \\ & & & \ddots & \\ w_{s 1}\left(\sigma_{s}\right) & \ldots & w_{s k}\left(\sigma_{s}\right) & \ldots & w_{s s}\left(\sigma_{s}\right)\end{array}\right]_{s \times s}$ is a weight matrix, where the matrix elements represent the tightness degree between adjacent PNs. If the PNs $\sigma_{i}$ and $\sigma_{k}$ are connected, then $w_{i k}\left(\sigma_{i}\right)$ is a weighted value in $[0,1]$ representing the tightness degree between $\sigma_{i}$ and $\sigma_{k}$; otherwise, $w_{i k}\left(\sigma_{i}\right)=0$, $1 \leq i, k \leq s$.

(7) $\mathbf{Y}_{k}^{-}=\left[\begin{array}{ccccc}y_{11}\left(\sigma_{1}\right) & \ldots & y_{1 k}\left(\sigma_{1}\right) & \ldots & y_{1 s}\left(\sigma_{1}\right) \\ & \ddots & & & \\ \vdots & & y_{i k}\left(\sigma_{i}\right) & & \vdots \\ & & & \ddots & \\ y_{s 1}\left(\sigma_{s}\right) & \ldots & y_{s k}\left(\sigma_{s}\right) & \ldots & y_{s s}\left(\sigma_{s}\right)\end{array}\right]_{s \times s} \quad$ is a danger degree matrix of PNs, where $y_{i k}\left(\sigma_{i}\right)$ is a number in $[0,1]$ representing the danger degree of the event corresponding to the $\mathrm{PN} \sigma_{i}$ triggers the one associated with the PN $\sigma_{k}, 1 \leq i, k \leq s$. If $\sigma_{i}$ can emit a spike to $\sigma_{k}$, then $y_{i k}\left(\sigma_{i}\right)$ is obtained via (3); otherwise, $y_{i k}\left(\sigma_{i}\right)=0$.

Note that the vectors $\boldsymbol{\lambda}_{p}, \boldsymbol{\lambda}_{r}, \boldsymbol{\alpha}, \boldsymbol{\delta}$, and $\mathbf{0}$, the matrices $\mathbf{D}_{1}, \mathbf{D}_{2}, \mathbf{D}_{3}$, and $\mathbf{D}_{4}$, and the operators $\otimes, \oplus$, and $\Delta$ in Algorithm 3 are the same as the ones in the Algorithms 1 and 2 . 


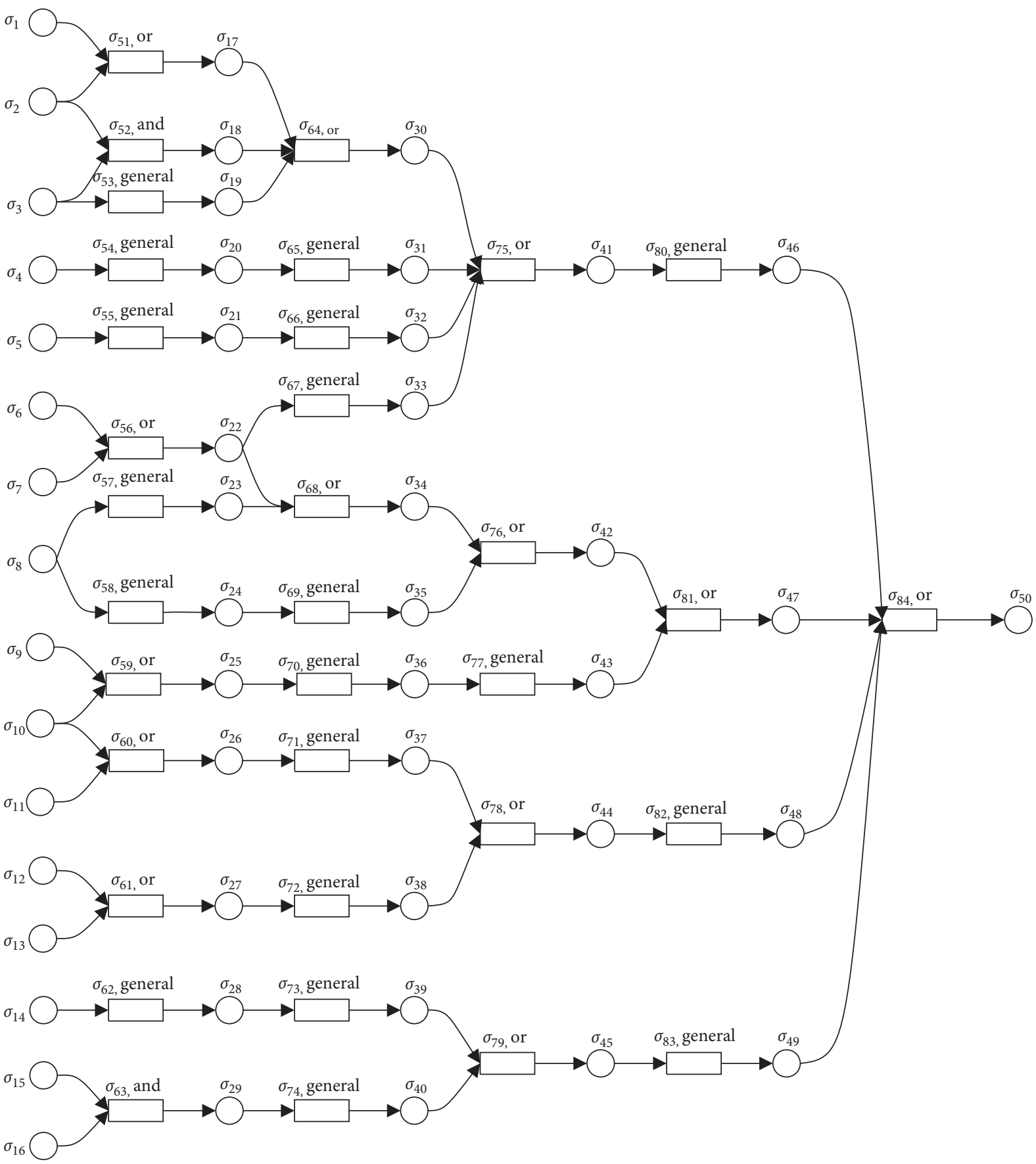

FIGURE 3: An rMFRSNPS-based model for three-phase induction motors.

\section{Case Studies}

In this section, several cases about possible faults on a motor are considered, in order to show the feasibility and validity of our proposed method. Note that the initial pulse values of input neurons in Algorithms 1 and 2 are the occurrence probabilities of fault symptoms obtained based on historical data and expertise. Since Algorithm 3 is used to find fault causes and fault sources after a motor fails, its initial pulse values are the event probabilities obtained by Algorithm 1, including the occurrence probabilities of both the fault symptoms and failures. 


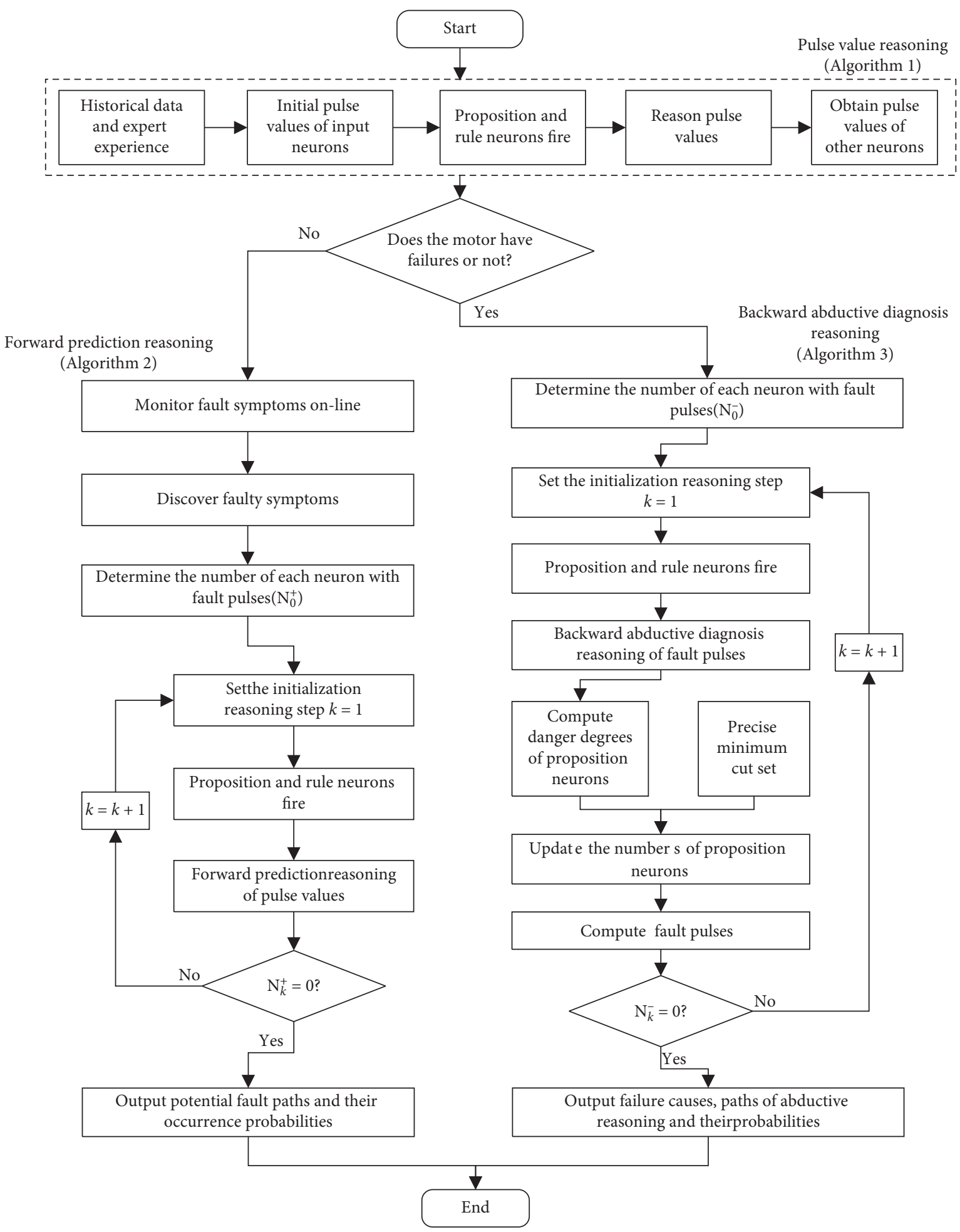

FIGURE 4: Fault reasoning flow chart based on rMFRSNPSs.

4.1. Pulse Value Reasoning of Neurons. The initial pulse value of input neurons and truth value of rule neurons are obtained via historical data and expert experience [23].

Here, we take the "insulation winding burned down" as an example. Then, we can get that the initial pulse value vectors of proposition neurons and rule neurons are $\boldsymbol{\alpha}_{0}=$ $\left(0.8,0.6,0.9,0.92,0.8,0.9,0.62,0.82, \mathbf{O}_{18}\right)^{T}, \delta_{0}=\left(\mathbf{O}_{18}\right)^{T}$, respectively.

The truth value diagonal matrix of $\mathrm{RNs}$ is $\mathbf{C}=\operatorname{diag}(0.8,0.88,0.87,0.8,0.92,0.89,0.89,0.89$, $0.89,0.92,0.94,0.9,0.92,0.91,0.94,0.93,0.97,0.93)$. 


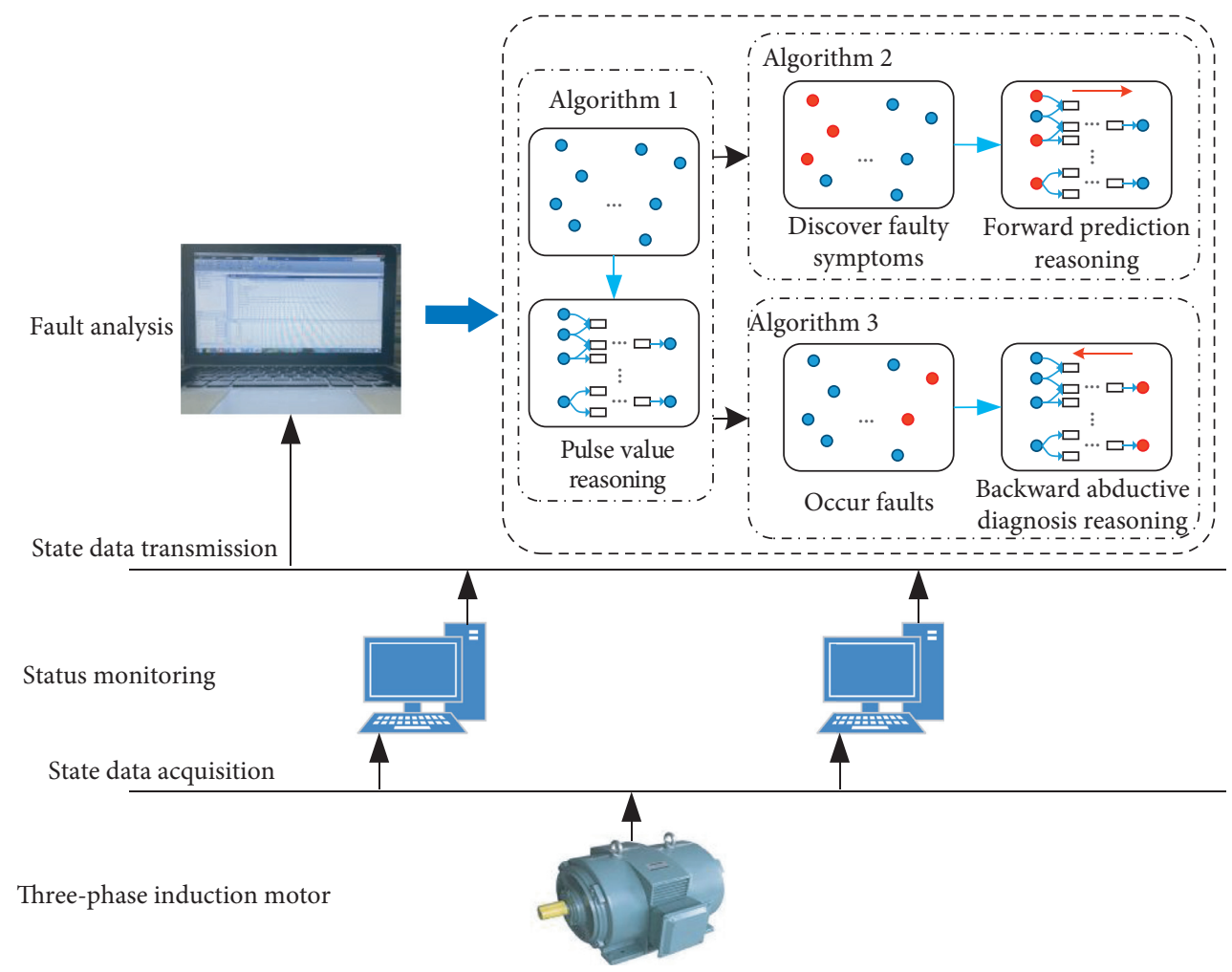

FIGURE 5: A diagrammatic sketch of the application scenario for the proposed method.

Input: $\boldsymbol{a}_{0}, \boldsymbol{\delta}_{0}, \mathbf{D}_{1}, \mathbf{D}_{2}, \mathbf{D}_{3}, \mathbf{D}_{4}, \mathbf{C}, \boldsymbol{\lambda}_{p}, \boldsymbol{\lambda}_{r}, \mathbf{0}=(0, \ldots, 0)_{t}^{T}$

(1) Let $k=1$

(2) while $\left(\boldsymbol{\delta}_{k} \neq 0\right)$

(3) if each proposition neuron satisfies its firing condition $E=\left\{a^{n} \wedge \alpha_{i} \geq \lambda_{p_{i}}, 1 \leq i \leq s\right\}$ then

(4) proposition neurons fire and compute $\delta_{k}$ via $\boldsymbol{\delta}_{k}=\left(\mathbf{D}_{1}^{T} * \boldsymbol{\alpha}_{k-1}\right)+\left(\mathbf{D}_{2}^{T} \cdot \boldsymbol{\alpha}_{k-1}\right)+\left(\mathbf{D}_{3}^{T} \circ \boldsymbol{\alpha}_{k-1}\right)$

(5) if each rule neuron satisfies its firing condition

$$
E=\left\{a^{n} \wedge \delta_{j} \geq \lambda_{r_{j}}, 1 \leq j \leq t\right\} \text { then }
$$

(6) rule neurons fire and compute $\boldsymbol{\alpha}_{k}$ via

(7) end if

$$
\boldsymbol{\alpha}_{k}=\mathbf{D}_{4}^{T} \text { 。 }\left(\mathbf{C} * \boldsymbol{\delta}_{k}\right)
$$

(8) $k=k+1$

(9) end while

Output: The pulse value of all neurons.

Algorithm 1: Pulse value reasoning algorithm. 
Input: $\mathbf{N}_{p_{0}}^{+}, \mathbf{N}_{r_{0}}^{+}, \boldsymbol{\alpha}_{0}, \boldsymbol{\delta}_{0}, \boldsymbol{\lambda}_{p}, \boldsymbol{\lambda}_{r}, \mathbf{C}, \mathbf{0}=(0, \ldots, 0)_{t}^{T}, \mathbf{D}_{1}, \mathbf{D}_{2}, \mathbf{D}_{3}, \mathbf{D}_{4}$

(1) Let $k=1$

(2) while $\left(\mathbf{N}_{r_{k}}^{+} \neq 0\right)$

(3) if each proposition neuron satisfies its firing condition $E=\left\{a^{n} \wedge \alpha_{i} \geq \lambda_{p_{i}}, 1 \leq i \leq s\right\}$ then

(4) proposition neurons fire and compute $\boldsymbol{\delta}_{k}$ and $\mathbf{N}_{r_{k}}^{+}$via $\left\{\begin{array}{l}\boldsymbol{\delta}_{k}=\left(\mathbf{D}_{1}^{T} * \boldsymbol{\alpha}_{k-1}\right)+\left(\mathbf{D}_{2}^{T} \cdot \boldsymbol{\alpha}_{k-1}\right)+\left(\mathbf{D}_{3}^{T} \circ \boldsymbol{\alpha}_{k-1}\right), \\ \mathbf{N}_{r_{k}}^{+}=\left[\left(\mathbf{D}_{1} \oplus \mathbf{D}_{3}\right)^{T} \Delta\left(\mathbf{N}_{p_{k-1}^{+}}^{+} \otimes \boldsymbol{\alpha}_{k-1} \Theta \boldsymbol{\lambda}_{p}\right)\right] \oplus\left[\mathbf{D}_{2}^{T} \nabla\left(\mathbf{N}_{p_{k-1}}^{+} \otimes \boldsymbol{\alpha}_{k-1} \Theta \boldsymbol{\lambda}_{p}\right)\right]\end{array}\right.$

(5) end if

(6) if each rule neuron satisfies its firing condition

$$
E=\left\{a^{n} \wedge \delta_{j} \geq \lambda_{r_{j}}, 1 \leq j \leq t\right\} \text { then }
$$

(7) rule neurons fire and compute $\boldsymbol{\alpha}_{k}$ and $\mathbf{N}_{p_{k}}^{+}$via

$$
\left\{\begin{array}{l}
\boldsymbol{\alpha}_{k}=\mathbf{D}_{4}^{T} \circ\left(\mathbf{C} * \boldsymbol{\delta}_{k}\right), \\
\mathbf{N}_{p_{k}}^{+}=\mathbf{D}_{4}^{T} \Delta\left(\mathbf{N}_{r_{k}}^{+} \otimes \boldsymbol{\delta}_{k} \Theta \lambda_{r}\right)
\end{array}\right.
$$

(8) end if

(9) $k=k+1$

(10) end while

Output: Potential fault paths and their occurrence probabilities.

Algorithm 2: Forward fault prediction reasoning algorithm.

The firing threshold vectors for PNs and RNs are $\lambda_{p}=$ $(0.5, \ldots, 0.5)_{s \times 1}^{T}$ and $\lambda_{r}=(0.5, \ldots, 0.5)_{t \times 1}^{T}$, respectively.

The synaptic matrices $\mathbf{D}_{1}, \mathbf{D}_{2}, \mathbf{D}_{3}$, and $\mathbf{D}_{4}$ are obtained via the rMFRSNPS-based fault analysis model, as shown in Figure 3.

$$
\begin{aligned}
\mathbf{D}_{1} & =\left[\begin{array}{lllll}
\mathbf{V}_{7 \times 6} & \mathbf{O}_{7 \times 2} & \mathbf{O}_{7 \times 5} & \mathbf{O}_{7 \times 1} & \mathbf{O}_{7 \times 4} \\
\mathbf{O}_{1 \times 6} & \mathbf{E}_{1 \times 2} & \mathbf{O}_{1 \times 5} & \mathbf{O}_{1 \times 1} & \mathbf{O}_{1 \times 4} \\
\mathbf{O}_{7 \times 6} & \mathbf{O}_{7 \times 2} & \mathbf{V}_{7 \times 5} & \mathbf{O}_{7 \times 1} & \mathbf{O}_{7 \times 4} \\
\mathbf{O}_{1 \times 6} & \mathbf{O}_{1 \times 2} & \mathbf{O}_{1 \times 5} & \mathbf{E}_{1 \times 1} & \mathbf{O}_{1 \times 4} \\
\mathbf{O}_{10 \times 6} & \mathbf{O}_{10 \times 2} & \mathbf{O}_{10 \times 5} & \mathbf{O}_{10 \times 1} & \mathbf{V}_{10 \times 4}
\end{array}\right], \\
\mathbf{D}_{2} & =\left[\begin{array}{lll}
\mathbf{O}_{1 \times 1} & \mathbf{O}_{1 \times 1} & \mathbf{O}_{1 \times 16} \\
\mathbf{O}_{2 \times 1} & \mathbf{E}_{2 \times 1} & \mathbf{O}_{2 \times 16} \\
\mathbf{O}_{23 \times 1} & \mathbf{O}_{23 \times 1} & \mathbf{O}_{23 \times 16}
\end{array}\right], \\
\mathbf{D}_{3} & =\left[\begin{array}{lllll}
\mathbf{V}_{8 \times 6} & \mathbf{O}_{8 \times 3} & \mathbf{O}_{8 \times 3} & \mathbf{O}_{8 \times 3} & \mathbf{O}_{8 \times 3} \\
\mathbf{O}_{3 \times 6} & \mathbf{V}_{3 \times 3} & \mathbf{O}_{3 \times 3} & \mathbf{O}_{3 \times 3} & \mathbf{O}_{3 \times 3} \\
\mathbf{O}_{2 \times 6} & \mathbf{O}_{2 \times 3} & \mathbf{O}_{2 \times 3} & \mathbf{O}_{2 \times 3} & \mathbf{O}_{2 \times 3} \\
\mathbf{O}_{4 \times 6} & \mathbf{O}_{4 \times 3} & \mathbf{O}_{4 \times 3} & \mathbf{V}_{4 \times 3} & \mathbf{O}_{4 \times 3} \\
\mathbf{O}_{3 \times 6} & \mathbf{O}_{3 \times 3} & \mathbf{O}_{3 \times 3} & \mathbf{V}_{3 \times 3} & \mathbf{O}_{3 \times 3} \\
\mathbf{O}_{4 \times 6} & \mathbf{O}_{4 \times 3} & \mathbf{O}_{4 \times 3} & \mathbf{O}_{4 \times 3} & \mathbf{V}_{4 \times 3} \\
\mathbf{O}_{2 \times 6} & \mathbf{O}_{2 \times 3} & \mathbf{O}_{2 \times 3} & \mathbf{O}_{2 \times 3} & \mathbf{O}_{2 \times 3}
\end{array}\right], \\
\mathbf{D}_{4}= & {\left[\begin{array}{llll}
\mathbf{O}_{18 \times 8} & \mathbf{E}_{18 \times 18}
\end{array}\right], }
\end{aligned}
$$

where $\mathbf{V}_{7 \times 6}=\left[\begin{array}{lll}\mathbf{O}_{2 \times 2} & \mathbf{O}_{2 \times 3} & \mathbf{O}_{2 \times 2} \\ \mathbf{O}_{3 \times 2} & \mathbf{E}_{3 \times 3} & \mathbf{O}_{3 \times 2} \\ \mathbf{O}_{1 \times 2} & \mathbf{O}_{1 \times 3} & \mathbf{O}_{1 \times 2}\end{array}\right]^{T}, \mathbf{V}_{7 \times 5}=\left[\begin{array}{lll}\mathbf{O}_{1 \times 3} & \mathbf{O}_{1 \times 3} & \mathbf{O}_{1 \times 1} \\ \mathbf{O}_{3 \times 3} & \mathbf{E}_{3 \times 3} & \mathbf{O}_{3 \times 1} \\ \mathbf{O}_{1 \times 3} & \mathbf{O}_{1 \times 3} & \mathbf{O}_{1 \times 1}\end{array}\right]^{T}$, $\mathbf{V}_{10 \times 4}=\left[\begin{array}{lll}\mathbf{O}_{2 \times 6} & \mathbf{O}_{2 \times 1} & \mathbf{O}_{2 \times 3} \\ \mathbf{O}_{1 \times 6} & \mathbf{E}_{1 \times 1} & \mathbf{O}_{1 \times 3} \\ \mathbf{O}_{1 \times 6} & \mathbf{O}_{1 \times 1} & \mathbf{O}_{1 \times 3}\end{array}\right]^{T}, \mathbf{V}_{4 \times 3}=\left[\begin{array}{lll}\mathbf{E}_{1 \times 2} & \mathbf{O}_{1 \times 1} & \mathbf{O}_{1 \times 1} \\ \mathbf{O}_{1 \times 2} & \mathbf{O}_{1 \times 1} & \mathbf{O}_{1 \times 1} \\ \mathbf{O}_{1 \times 2} & \mathbf{O}_{1 \times 1} & \mathbf{E}_{1 \times 1}\end{array}\right]^{T}$, $\mathbf{V}_{3 \times 3}=\left[\begin{array}{l}\mathbf{O}_{2 \times 3} \\ \mathbf{E}_{1 \times 3}\end{array}\right]^{T}, \mathbf{V}_{8 \times 6}=\left[\begin{array}{llll}\mathbf{E}_{1 \times 2} & \mathbf{O}_{1 \times 3} & \mathbf{O}_{1 \times 2} & \mathbf{O}_{1 \times 1} \\ \mathbf{O}_{4 \times 2} & \mathbf{O}_{4 \times 3} & \mathbf{O}_{4 \times 2} & \mathbf{O}_{4 \times 1} \\ \mathbf{O}_{1 \times 2} & \mathbf{O}_{1 \times 3} & \mathbf{E}_{1 \times 2} & \mathbf{O}_{1 \times 1}\end{array}\right]^{T}, \mathbf{O}$ is a null matrix, and $\mathbf{E}$ is an identity matrix.

The pulse value reasoning process is described as follows:

$$
\begin{aligned}
& \text { When } k=1, \quad \boldsymbol{\delta}_{1}=(0.8,0.6,0.9,0.92,0.8,0.9,0.62, \\
& \left.0.82, \mathbf{O}_{10}\right)^{T}, \quad \boldsymbol{\alpha}_{1}=\left(\mathbf{O}_{8}, 0.64,0.53,0.78,0.74,0.74,0.8,\right. \\
& \left.0.73,0.73, \mathbf{O}_{10}\right)^{T} \\
& \text { When } k=2, \quad \boldsymbol{\delta}_{2}=\left(\mathbf{O}_{8}, 0.78,0.74,0.74,0.8,0.8,0.73,\right. \\
& \left.\mathbf{O}_{4}\right)^{T}, \boldsymbol{\alpha}_{2}=\left(\mathbf{O}_{16}, 0.69,0.68,0.7,0.72,0.74,0.66, \mathbf{O}_{4}\right)^{T} \\
& \text { When } k=3, \quad \boldsymbol{\delta}_{3}=\left(\mathbf{O}_{14}, 0.72,0.74, \mathbf{O}_{2}\right)^{T}, \quad \boldsymbol{\alpha}_{3}=\left(\mathbf{O}_{22},\right. \\
& \text { 0.68, 0.69, } \left.\mathbf{O}_{2}\right)^{T} \\
& \text { When } k=4, \quad \boldsymbol{\delta}_{4}=\left(\mathbf{O}_{16}, 0.68,0.69\right)^{T}, \boldsymbol{\alpha}_{4}=\left(\mathbf{O}_{24}, 0.66,\right. \\
& \text { 0.64 })^{T} \\
& \text { When } k=5, \boldsymbol{\delta}_{5}=\left(\mathbf{O}_{18}\right)^{T}
\end{aligned}
$$

Thus, the termination condition is satisfied and the reasoning stops. We obtain the reasoning results, i.e., the pulse value of all neurons, shown as follows: 
Input: $\mathbf{N}_{p_{0}}^{-}, \mathbf{N}_{r_{0}}^{-}, \boldsymbol{\theta}_{p_{0}}, \boldsymbol{\theta}_{r_{0}}, \boldsymbol{\alpha}, \boldsymbol{\delta}, \boldsymbol{\lambda}_{p}, \boldsymbol{\lambda}_{r}, \lambda_{y}, \mathbf{0}=(0, \ldots, 0)_{t}^{T}, \mathbf{D}_{1}, \mathbf{D}_{2}, \mathbf{D}_{3}, \mathbf{D}_{4}, \mathbf{W}$

(1) Let $k=1$

(2) while $\left(\mathbf{N}_{r}^{-} \neq 0\right)$

(3) if each rule neurons satisfies its firing condition $E=\left\{a^{n} \wedge \theta_{j} \geq \lambda_{r_{j}}, 1 \leq j \leq t\right\}$ then

(4) rule neurons fire and compute $\mathbf{N}_{r_{k}}^{-}$and $\boldsymbol{\theta}_{r_{k}}$ via

$$
\left\{\begin{array}{l}
\mathbf{N}_{r_{k}}^{-}=\mathbf{D}_{4} \Delta \mathbf{N}_{p_{k-1}}^{-} \\
\boldsymbol{\theta}_{r_{k}}=\mathbf{N}_{r_{k}}^{-} \otimes \boldsymbol{\delta}
\end{array}\right.
$$

(5) end if

(6) if each proposition neuron satisfies its firing condition $E=\left\{a^{n} \wedge \theta_{i} \geq \lambda_{p_{i}}, 1 \leq i \leq s\right\}$ then

(7) proposition neurons fire and compute $\mathbf{N}_{p_{k}}^{-}$and $\boldsymbol{\theta}_{p_{k}}$ via

$$
\left\{\begin{array}{l}
\mathbf{N}_{p_{k}}^{-}=\left(\mathbf{D}_{1} \oplus \mathbf{D}_{2} \oplus \mathbf{D}_{3}\right) \Delta \mathbf{N}_{r_{k}}^{-} \\
\boldsymbol{\theta}_{p_{k}}=\mathbf{N}_{p_{k}}^{-} \otimes \mathbf{a}
\end{array}\right.
$$

(8) compute $\mathbf{Y}_{k}^{-}$via

$$
\mathbf{Y}_{k}^{-}=\mathbf{W} \otimes \boldsymbol{\theta}_{p_{k}}
$$

(9) determine the MCSs $\left(Q_{1}, \ldots, Q_{l}\right)$ of each PN in $\mathbf{N}_{p_{k-1}}^{-}$, where $Q_{i}=\left(\sigma_{1}, \ldots, \sigma_{q}\right), 1 \leq q, i<s$. Compute danger degree of MCSs for each PN via $y\left(Q_{i}\right)=\prod_{j=1}^{q} y\left(\sigma_{j}\right)$, and screen out the pulse of PNs in a PMCS whose danger degree is larger than $\lambda_{y}$

(10) update the number of propositional neurons $\mathbf{N}_{p_{k}}^{-}$per the selected pulses, and compute pulse value of fault pulse in proposition neurons after position updating via

(11) end if

$$
\boldsymbol{\theta}_{p_{k}}=\mathbf{N}_{p_{k}}^{-} \otimes \boldsymbol{a}
$$

(12) $k=k+1$

(13) end while

Output: Failure causes, paths of abductive reasoning and their probabilities.

Algorithm 3: Backward abductive fault diagnosis reasoning algorithm.

$\boldsymbol{\alpha}=(0.8,0.6,0.9,0.92,0.8,0.9,0.62,0.82,0.64,0.53,0.78,0.74,0.74,0.8,0.73,0.73,0.69,0.68,0.7,0.72,0.74,0.66,0.68,0.69,0.66,0.64)^{T}$, $\boldsymbol{\delta}=(0.8,0.6,0.9,0.92,0.8,0.9,0.62,0.82,0.78,0.74,0.74,0.8,0.8,0.73,0.72,0.74,0.68,0.69)^{T}$.

4.2. Forward Fault Prediction Reasoning. Let us assume that the following fault symptoms of a motor are monitored online: overload $(p 1)$, resistance value of a phase winding decreases ( $p 3)$, damage of shaft seal ring structure $(p 5)$, and excessive roughness value of seal surface shaft $(p 7)$. Accordingly, the initial number vector $\mathbf{N}_{p_{0}}^{+}$of the PNs with fault pulses is obtained: $\mathbf{N}_{p_{0}}^{+}=\left(1,0,1,0,1,0,1, \mathbf{O}_{19}\right)^{T}$.

The synaptic matrices $\mathbf{D}_{1}, \mathbf{D}_{2}, \mathbf{D}_{3}$, and $\mathbf{D}_{4}$ are the same as the ones in Section 4.1.

The initial pulse value vectors of PNs and RNs are $\boldsymbol{\alpha}_{0}=$ $\left(0.8,0.6,0.9,0.92,0.8,0.9,0.62,0.82, \mathbf{O}_{18}\right)^{T}, \delta_{0}=\left(\mathbf{O}_{18}\right)^{T}$, respectively.

The truth value diagonal matrix of $\mathrm{RNs}$ is $\mathbf{C}=\operatorname{diag}(0.8,0.88,0.87,0.8,0.92,0.89,0.89,0.89$, $0.89,0.92,0.94,0.9,0.92,0.91,0.94,0.93,0.97,0.93)$.

The fault prediction reasoning process is described as follows:

When $\quad k=1, \quad \boldsymbol{\delta}_{1}=(0.8,0.6,0.9,0.92,0.8,0.9,0.62$, $\left.0.82, \mathbf{O}_{10}\right)^{T}, \mathbf{N}_{r_{1}}^{+}=\left(1,0,1,0,1,1, \mathbf{O}_{12}\right)^{T} \cdot \boldsymbol{\alpha}_{1}=\left(\mathbf{O}_{8}, 0.64\right.$, $\left.0.53,0.78,0.74,0.74,0.8,0.73,0.73, \mathbf{O}_{10}\right)^{T}, \mathbf{N}_{p_{1}}^{+}=\left(\mathbf{O}_{8} 1\right.$, $\left.0,1,0,1,1, \mathbf{O}_{12}\right)^{T}$.
When $k=2, \quad \boldsymbol{\delta}_{2}=\left(\mathbf{O}_{8}, 0.78,0.74,0.74,0.8,0.8,0.73\right.$, $\left.\mathbf{O}_{4}\right)^{T}, \quad \mathbf{N}_{r_{2}}^{+}=\left(\mathbf{O}_{8}, 1,0,1,1,1, \mathbf{O}_{5}\right)^{T} . \quad \boldsymbol{\alpha}_{2}=\left(\mathbf{O}_{16}, 0.69\right.$, $\left.0.68,0.7,0.72,0.74,0.66, \mathbf{O}_{4}\right)^{T}, \quad \mathbf{N}_{p_{2}}^{+}=\left(\mathbf{O}_{16} 1, \quad 0,1,0\right.$, $\left.1,11, \mathbf{O}_{5}\right)^{T}$.

When $k=3, \boldsymbol{\delta}_{3}=\left(\mathbf{O}_{14}, 0.72,0.74, \mathbf{O}_{2}\right)^{T}, \mathbf{N}_{r_{3}}^{+}=\left(\mathbf{O}_{14}\right.$, $\left.1,1, \mathbf{O}_{2}\right)^{T} ; \quad \boldsymbol{\alpha}_{3}=\left(\mathbf{O}_{22}, 0.68,0.69, \mathbf{O}_{2}\right)^{T}, \quad \mathbf{N}_{p_{3}}^{+} \stackrel{\mathbf{P}_{3}}{=}\left(\mathbf{O}_{22}, 1\right.$, $\left.1, \mathbf{O}_{2}\right)^{T}$.

When $k=4, \boldsymbol{\delta}_{4}=\left(\mathbf{O}_{16}, 0.68,0.69\right)^{T}, \mathbf{N}_{r_{4}}^{+}=\left(\mathbf{O}_{15}, 1,1\right)^{T}$. $\boldsymbol{\alpha}_{4}=\left(\mathbf{O}_{24}, 0.66,0.64\right)^{T}, \mathbf{N}_{p_{4}}^{+}=\left(\mathbf{O}_{24} 1,1\right)^{T}$.

When $k=5, \boldsymbol{\delta}_{5}=\left(\mathbf{O}_{18}\right)^{T}, \mathbf{N}_{r_{5}}^{+}=\left(\mathbf{O}_{18}\right)^{T}$.

Thus, the termination condition is satisfied and the reasoning stops. We find that the neurons with fault pulses are shown in Figure 6. Therefore, the potential fault paths are obtained; that is, $L_{1}=\left(\sigma_{1}, \sigma_{17}, \sigma_{30}, \sigma_{41}, \sigma_{46}\right)$, $L_{2}=\left(\sigma_{3}, \sigma_{19}, \sigma_{30}, \sigma_{41}, \sigma_{46}\right), \quad L_{3}=\left(\sigma_{5}, \sigma_{21}, \sigma_{32}, \sigma_{41}, \sigma_{46}\right)$, $L_{4}=\left(\sigma_{7}, \sigma_{22}, \sigma_{33}, \sigma_{41}, \sigma_{46}\right)$, and $L_{5}=\left(\sigma_{7}, \sigma_{22}, \sigma_{34}, \sigma_{42}, \sigma_{47}\right)$, where $\sigma_{17}, \sigma_{19}, \sigma_{21}, \sigma_{22}, \sigma_{30}, \sigma_{32}, \sigma_{33}, \sigma_{34}, \sigma_{41}, \sigma_{42}, \sigma_{46}$, and $\sigma_{47}$ are potential faults. The occurrence probability of each fault path is $P\left(L_{1}\right)=0.159, P\left(L_{2}\right)=0.217, P\left(L_{3}\right)=0.186$, $P\left(L_{4}\right)=0.16$, and $P\left(L_{5}\right)=0.162$, respectively. Thus, the checking order of the fault paths is $L_{2}, L_{3}, L_{5}, L_{4}, L_{1}$. Note that 


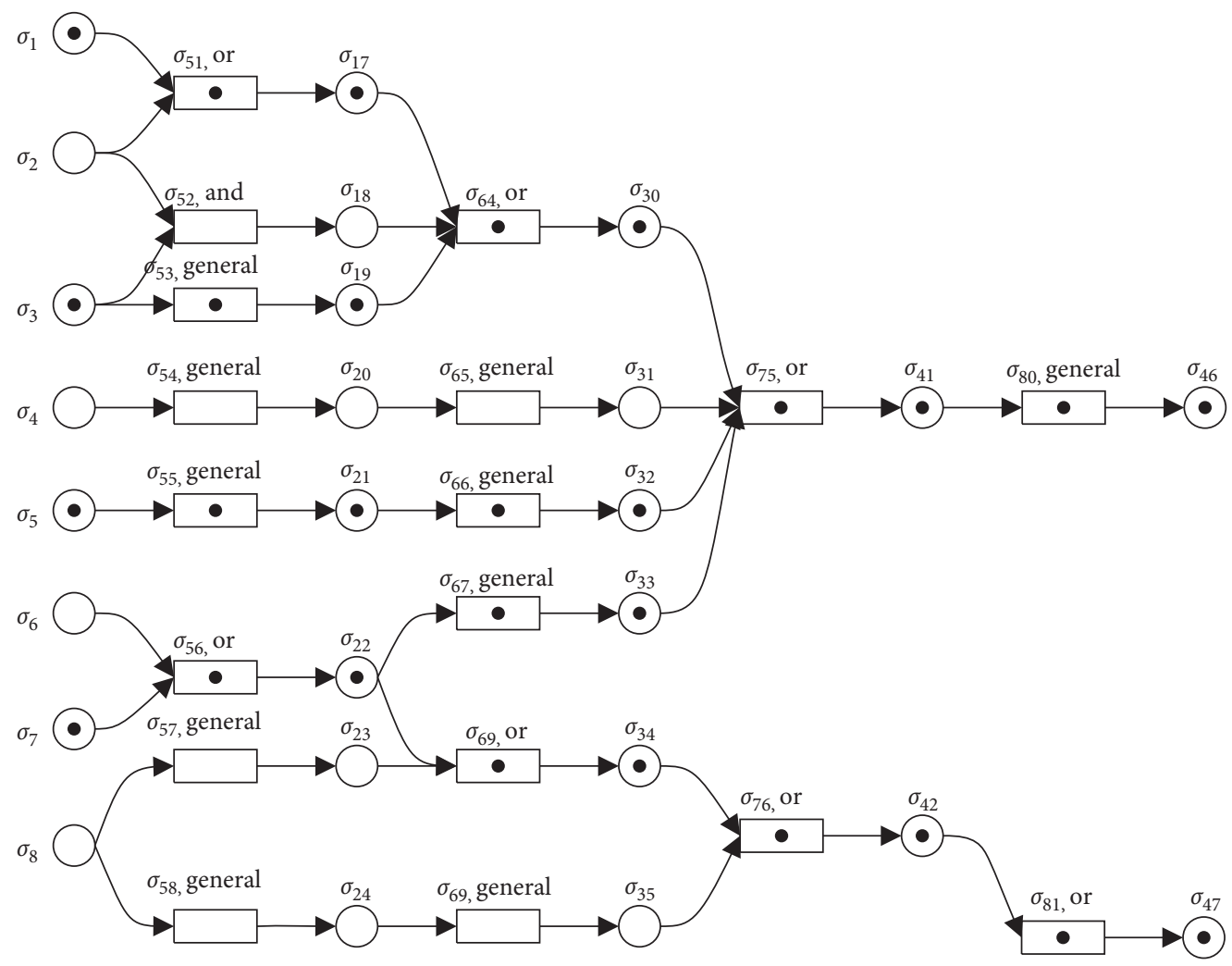

FIGURE 6: Forward prediction of potential fault paths.

the probability of a path is obtained by multiplying the pulse value of the neurons in each path.

4.3. Backward Abductive Fault Diagnosis Reasoning. This section assumes that the motor has failures. Let us take insulation winding burned down ( $p 46)$ as an example. Accordingly, the number vector of PNs with fault pulse is $\mathbf{N}_{p_{0}}^{-}=\left(\mathbf{O}_{24}, 1, \mathbf{O}_{1}\right)^{T}$.

The pulse value vectors of PNs and RNs are $\alpha$ and $\delta$, respectively, given as follows:

$\boldsymbol{\alpha}=(0.8,0.6,0.9,0.92,0.8,0.9,0.62,0.82,0.64,0.53,0.78,0.74,0.74,0.8,0.73,0.73,0.69,0.68,0.7,0.72,0.74,0.66,0.68,0.69,0.66,0.64)^{T}$,

$\boldsymbol{\delta}=(0.8,0.6,0.9,0.92,0.8,0.9,0.62,0.82,0.78,0.74,0.74,0.8,0.8,0.73,0.72,0.74,0.68,0.69)^{T}$.

The synaptic matrices $\mathbf{D}_{1}, \mathbf{D}_{2}, \mathbf{D}_{3}$, and $\mathbf{D}_{4}$ are the same as those in Section 4.1.

The tightness degree between PNs is shown in Figure 7, from which the weight matrix $\mathbf{W}$ can be obtained.

The abductive fault diagnosis reasoning process is described as follows:

When $k=1$,

$$
\begin{aligned}
\mathbf{N}_{r_{1}}^{-} & =\left(\mathbf{O}_{16}, 1, \mathbf{O}_{1}\right)^{T}, \\
\boldsymbol{\theta}_{r_{1}} & =\left(\mathbf{O}_{16}, 0.68, \mathbf{O}_{1}\right)^{T}, \\
\mathbf{N}_{p_{1}}^{-} & =\left(\mathbf{O}_{22}, 1, \mathbf{O}_{3}\right)^{T}, \\
\boldsymbol{\theta}_{p_{1}} & =\left(\mathbf{O}_{22}, 0.68, \mathbf{O}_{3}\right)^{T}, \\
\mathbf{Y}_{1}^{-} & =\left[\begin{array}{lll}
\mathbf{O}_{24 \times 22} & \mathbf{O}_{24 \times 1} & \mathbf{O}_{24 \times 3} \\
\mathbf{O}_{1 \times 22} & 0.68 & \mathbf{O}_{1 \times 3} \\
\mathbf{O}_{1 \times 22} & \mathbf{O}_{1 \times 1} & \mathbf{O}_{1 \times 3}
\end{array}\right]^{T}, \\
Q_{1} & =\left\{\sigma_{41}\right\}, \\
y\left(Q_{1}\right) & =y\left(\sigma_{41}\right)=0.68 .
\end{aligned}
$$




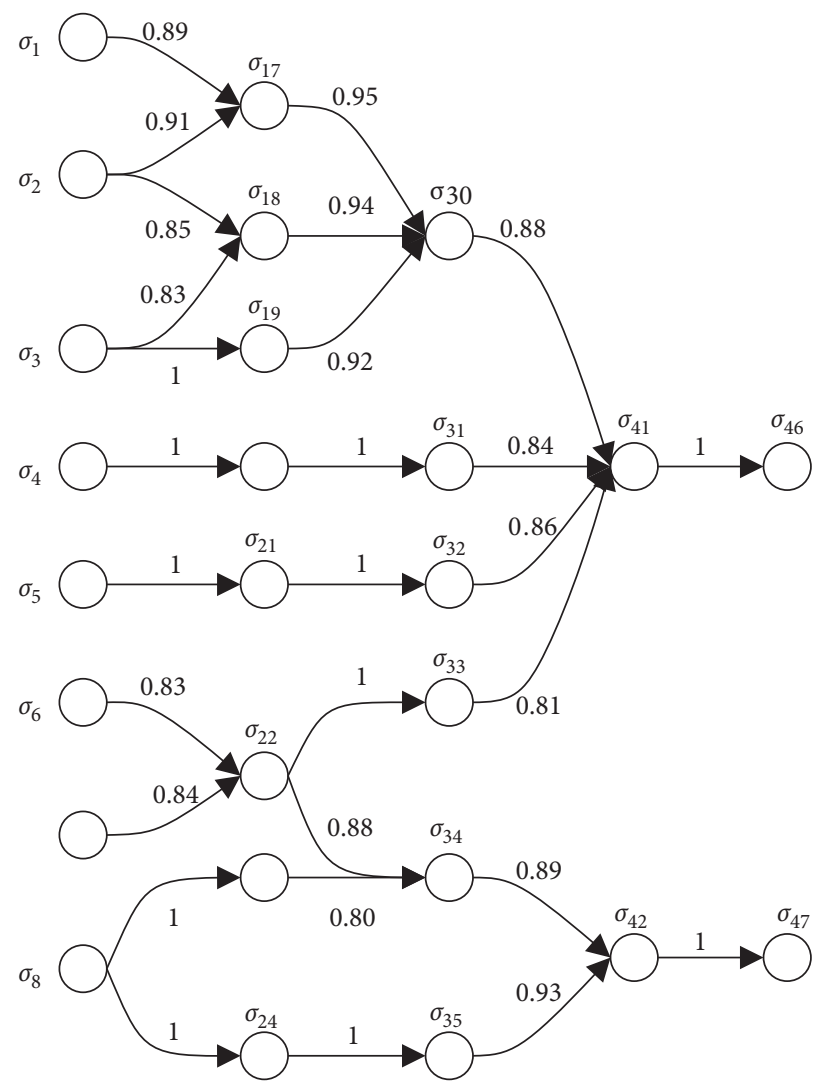

FIgUre 7: The tightness degree among PNs.

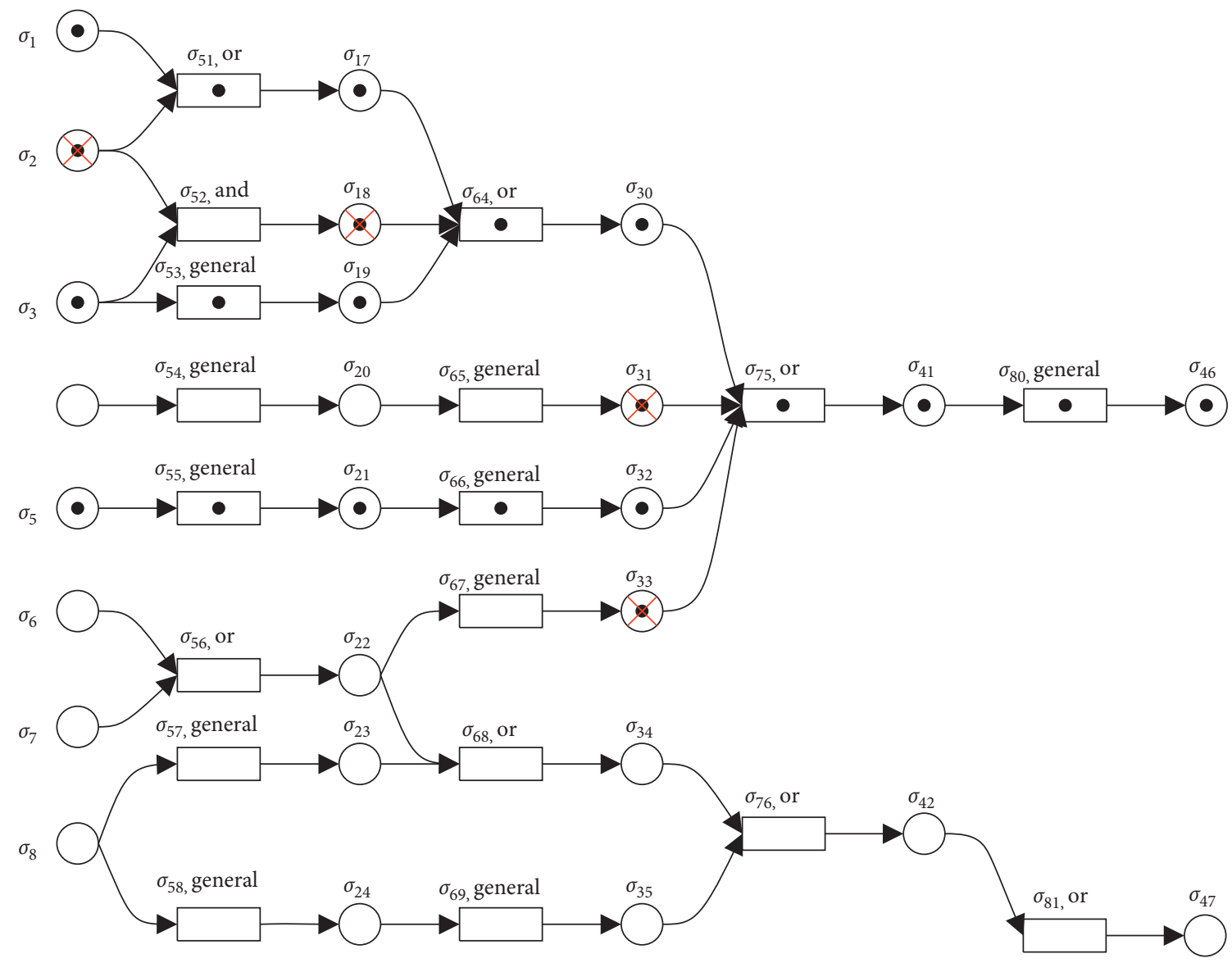

FIgURE 8: Backward fault abductive paths. 
TABLE 2: Results comparison of the rMFRSNPS and four other methods.

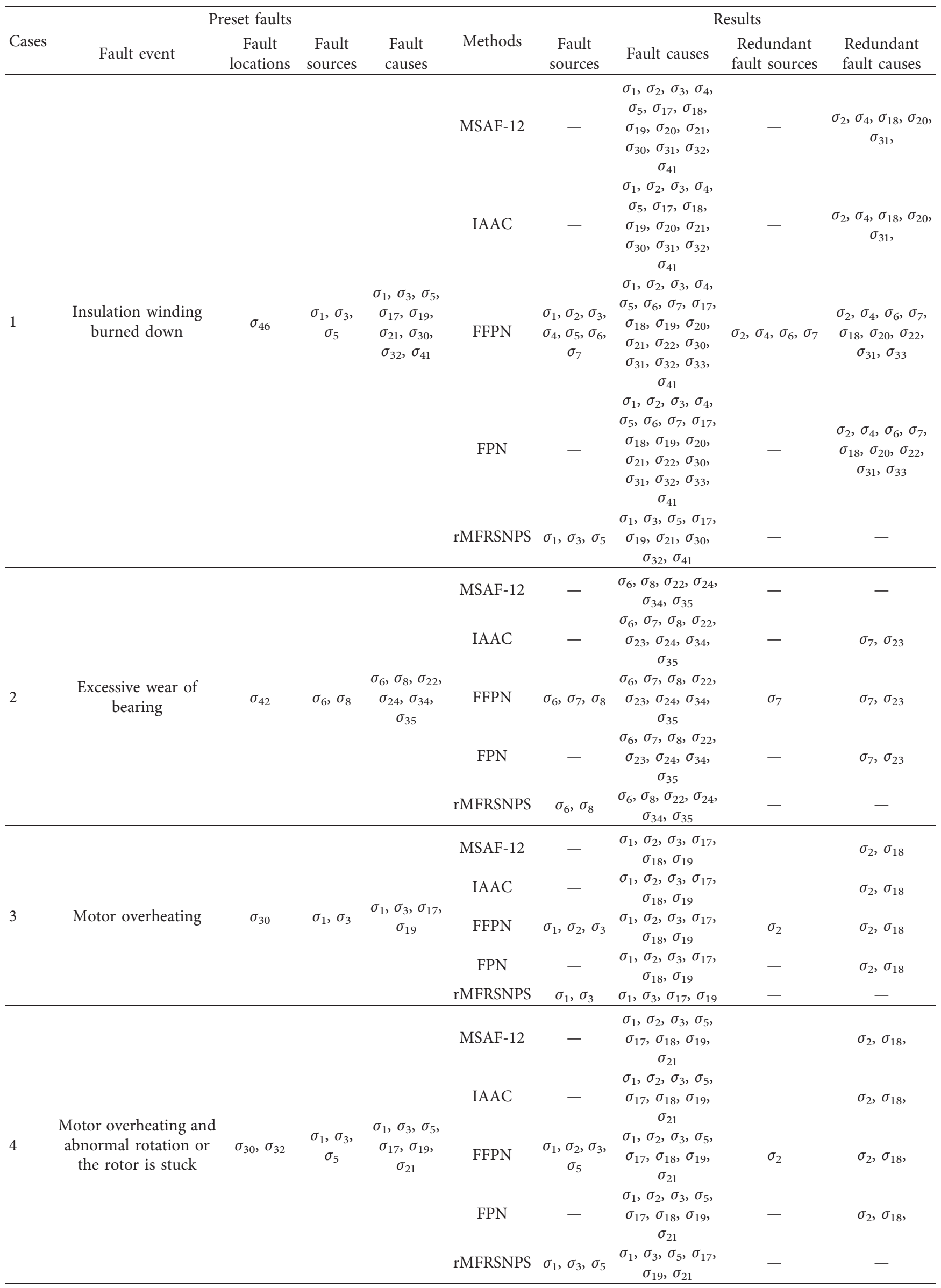


TABLE 2: Continued.

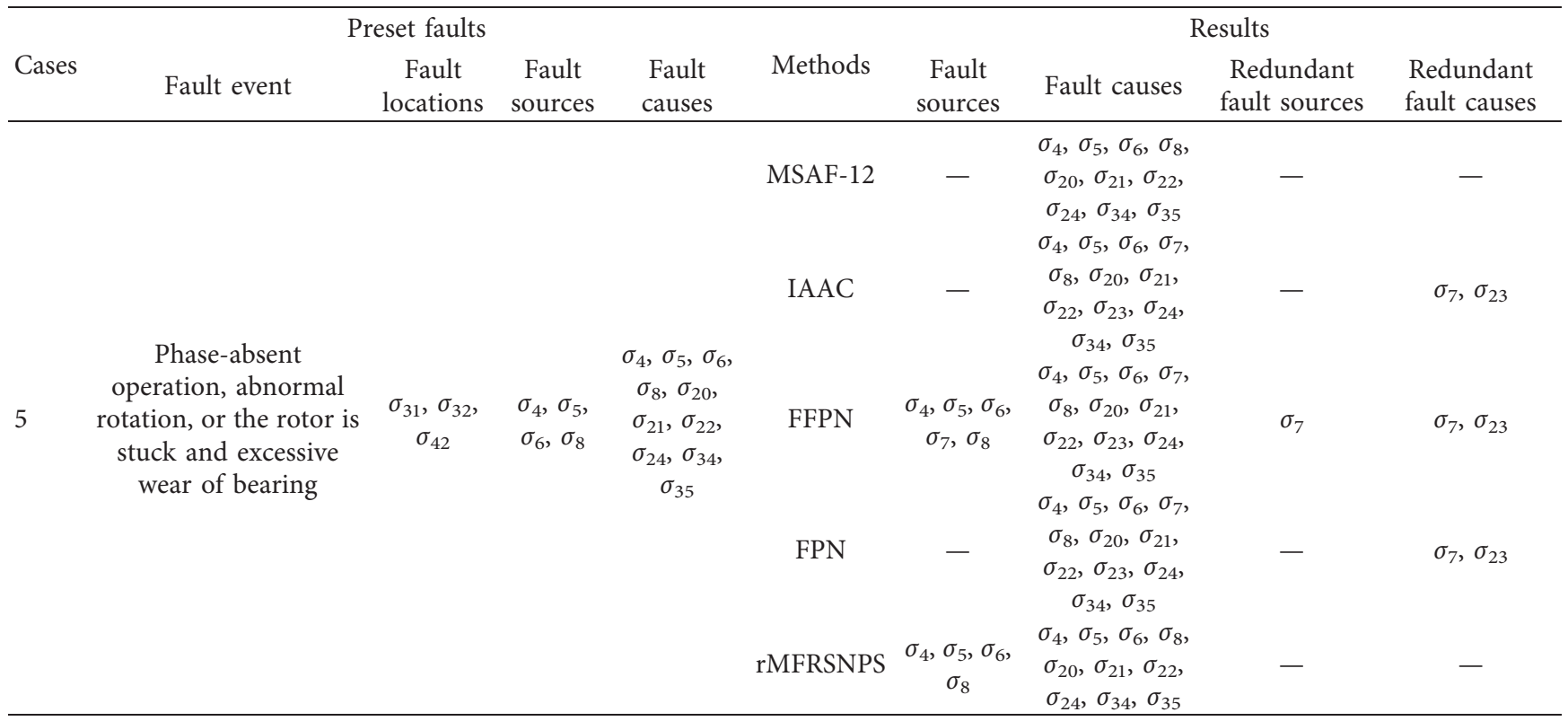

Note: "-" means that there is no such kind of information.

To start with the process, the pulse of PNs in the MCS whose danger degree is less than $\lambda_{y}$ is deleted. Accordingly, the number vectors of PNs and their corresponding pulse values of fault pulses are updated, i.e., $\mathbf{N}_{p_{1}}^{-}=\left(\mathbf{O}_{22}, 1, \mathbf{O}_{3}\right)^{T}$ and $\boldsymbol{\theta}_{p_{1}}=\left(\mathbf{O}_{22}, 0.68, \mathbf{O}_{3}\right)^{T}$, respectively.

When $k=2$,

$$
\begin{aligned}
& \mathbf{N}_{r_{2}}^{-}=\left(\mathbf{O}_{14}, 1, \mathbf{O}_{3}\right)^{T} \text {, } \\
& \boldsymbol{\theta}_{r_{2}}=\left(\mathbf{O}_{14}, 0.72, \mathbf{O}_{3}\right)^{T} \text {, } \\
& \mathbf{N}_{p_{2}}^{-}=\left(\mathbf{O}_{16}, 1,1,1,1, \mathbf{O}_{6}\right)^{T} \text {, } \\
& \boldsymbol{\theta}_{p_{2}}=\left(\mathbf{O}_{16}, 0.69,0.68,0.7,0.72, \mathbf{O}_{6}\right)^{T} \text {, } \\
& \mathbf{Y}_{2}^{-}=\left[\begin{array}{cccccc}
\mathbf{O}_{22 \times 16} & \mathbf{O}_{22 \times 1} & \mathbf{O}_{22 \times 1} & \mathbf{O}_{22 \times 1} & \mathbf{O}_{22 \times 1} & \mathbf{O}_{22 \times 6} \\
\mathbf{O}_{1 \times 16} & 0.61 & 0.57 & 0.6 & 0.58 & \mathbf{O}_{1 \times 6} \\
\mathbf{O}_{3 \times 16} & \mathbf{O}_{3 \times 1} & \mathbf{O}_{3 \times 1} & \mathbf{O}_{3 \times 1} & \mathbf{O}_{3 \times 1} & \mathbf{O}_{3 \times 6}
\end{array}\right]^{T}, \\
& Q_{1}=\left\{\sigma_{30}\right\} \text {, } \\
& Q_{2}=\left\{\sigma_{31}\right\} \text {, } \\
& Q_{3}=\left\{\sigma_{32}\right\} \text {, } \\
& Q_{4}=\left\{\sigma_{33}\right\} \text {, } \\
& y\left(Q_{1}\right)=y\left(\sigma_{30}\right)=0.61 \text {, } \\
& y\left(Q_{2}\right)=y\left(\sigma_{31}\right)=0.57 \text {, } \\
& y\left(Q_{3}\right)=y\left(\sigma_{32}\right)=0.6 \text {, } \\
& y\left(Q_{4}\right)=y\left(\sigma_{33}\right)=0.58 \text {. }
\end{aligned}
$$

Similarly, the pulse of PNs in the MCS whose danger degree is less than $\lambda_{y}$ must also be deleted. Accordingly, the number vectors of PNs and their corresponding pulse values of fault pulses are updated, i.e., $\mathbf{N}_{p_{2}}^{-}=\left(\mathbf{O}_{16}, 1,0,1, \mathbf{O}_{7}\right)^{T}, \boldsymbol{\theta}_{p_{2}}=\left(\mathbf{O}_{16}, 0.69,0,0.7, \mathbf{O}_{7}\right)^{T}$.

When $k=3$,

$$
\begin{aligned}
& \mathbf{N}_{r_{3}}^{-}=\left(\mathbf{O}_{8}, 1,0,1, \mathbf{O}_{7}\right)^{T}, \\
& \boldsymbol{\theta}_{r_{3}}=\left(\mathbf{O}_{8}, 0.78,0,0.74, \mathbf{O}_{7}\right)^{T}, \\
& \mathbf{N}_{p_{3}}^{-}=\left(\mathbf{O}_{8}, 1,1,1,0,1, \mathbf{O}_{13}\right)^{T} \text {, } \\
& \boldsymbol{\theta}_{p_{3}}=\left(\mathbf{O}_{8}, 0.64,0.53,0.78,0,0.74, \mathbf{O}_{13}\right)^{T} \text {, } \\
& \mathbf{Y}_{3}^{-}=\left[\begin{array}{ccccccc}
\mathbf{O}_{16 \times 8} & \mathbf{O}_{16 \times 1} & \mathbf{O}_{16 \times 1} & \mathbf{O}_{16 \times 1} & \mathbf{O}_{16 \times 1} & \mathbf{O}_{16 \times 1} & \mathbf{O}_{16 \times 13} \\
\mathbf{O}_{1 \times 8} & 0.61 & 0.5 & 0.72 & \mathbf{O}_{1 \times 1} & \mathbf{O}_{1 \times 1} & \mathbf{O}_{1 \times 13} \\
\mathbf{O}_{1 \times 8} & \mathbf{O}_{1 \times 1} & \mathbf{O}_{1 \times 1} & \mathbf{O}_{1 \times 1} & \mathbf{O}_{1 \times 1} & \mathbf{O}_{1 \times 1} & \mathbf{O}_{1 \times 13} \\
\mathbf{O}_{1 \times 8} & \mathbf{O}_{1 \times 1} & \mathbf{O}_{1 \times 1} & \mathbf{O}_{1 \times 1} & \mathbf{O}_{1 \times 1} & 0.74 & \mathbf{O}_{1 \times 13} \\
\mathbf{O}_{7 \times 8} & \mathbf{O}_{7 \times 1} & \mathbf{O}_{7 \times 1} & \mathbf{O}_{7 \times 1} & \mathbf{O}_{7 \times 1} & \mathbf{O}_{7 \times 1} & \mathbf{O}_{7 \times 13}
\end{array}\right]^{T}, \\
& Q_{1}=\left\{\sigma_{17}\right\} \text {, } \\
& Q_{2}=\left\{\sigma_{18}\right\} \text {, } \\
& Q_{3}=\left\{\sigma_{19}\right\} \text {, } \\
& Q_{4}=\left\{\sigma_{21}\right\} \text {, } \\
& y\left(Q_{1}\right)=y\left(\sigma_{17}\right)=0.61 \text {, } \\
& y\left(Q_{2}\right)=y\left(\sigma_{18}\right)=0.5 \text {, } \\
& y\left(Q_{3}\right)=y\left(\sigma_{19}\right)=0.72 \text {, } \\
& y\left(Q_{4}\right)=y\left(\sigma_{21}\right)=0.74 \text {. }
\end{aligned}
$$

Repeatedly, it must be made sure to delete the pulse of PNs in the MCS whose danger degree is less than $\lambda_{y}$. Accordingly, the number vectors of PNs and their corresponding pulse values of fault pulses are updated, 


$$
\begin{aligned}
& \text { i.e., } \quad \mathbf{N}_{p_{3}}^{-}=\left(\mathbf{O}_{8}, 1,0,1,0,1, \mathbf{O}_{13}\right)^{T} \quad \text { and } \\
& \boldsymbol{\theta}_{p_{3}}=\left(\mathbf{O}_{8}, 0.64,0,0.78,0,0.74, \mathbf{O}_{13}\right)^{T} \text {, respectively. } \\
& \text { When } k=4 \text {, } \\
& \mathbf{N}_{r_{4}}^{-}=\left(1,0,1,0,1, \mathbf{O}_{13}\right)^{T}, \\
& \boldsymbol{\theta}_{r_{4}}=\left(0.8,0,0.9,0,0.8, \mathbf{O}_{13}\right)^{T}, \\
& \mathbf{N}_{p_{4}}^{-}=\left(1,1,1,0,1, \mathbf{O}_{21}\right)^{T} \text {, } \\
& \boldsymbol{\theta}_{p_{4}}=\left(0.8,0.6,0.9,0,0.8, \mathbf{O}_{21}\right)^{T} \text {, } \\
& \mathbf{Y}_{4}^{-}=\left[\begin{array}{cccccc}
\mathbf{O}_{8 \times 1} & \mathbf{O}_{8 \times 1} & \mathbf{O}_{8 \times 1} & \mathbf{O}_{8 \times 1} & \mathbf{O}_{8 \times 1} & \mathbf{O}_{8 \times 21} \\
0.71 & 0.55 & \mathbf{O}_{1 \times 1} & \mathbf{O}_{1 \times 1} & \mathbf{O}_{1 \times 1} & \mathbf{O}_{1 \times 21} \\
\mathbf{O}_{1 \times 1} & 0.51 & 0.75 & \mathbf{O}_{1 \times 1} & \mathbf{O}_{1 \times 1} & \mathbf{O}_{1 \times 21} \\
\mathbf{O}_{1 \times 1} & \mathbf{O}_{1 \times 1} & 0.9 & \mathbf{O}_{1 \times 1} & \mathbf{O}_{1 \times 1} & \mathbf{O}_{1 \times 21} \\
\mathbf{O}_{1 \times 1} & \mathbf{O}_{1 \times 1} & \mathbf{O}_{1 \times 1} & \mathbf{O}_{1 \times 1} & \mathbf{O}_{1 \times 1} & \mathbf{O}_{1 \times 21} \\
\mathbf{O}_{1 \times 1} & \mathbf{O}_{1 \times 1} & \mathbf{O}_{1 \times 1} & \mathbf{O}_{1 \times 1} & 0.8 & \mathbf{O}_{1 \times 21} \\
\mathbf{O}_{13 \times 1} & \mathbf{O}_{13 \times 1} & \mathbf{O}_{13 \times 1} & \mathbf{O}_{13 \times 1} & \mathbf{O}_{13 \times 1} & \mathbf{O}_{13 \times 21}
\end{array}\right], \\
& Q_{1}=\left\{\sigma_{1}\right\} \text {, } \\
& Q_{2}=\left\{\sigma_{2}\right\} \text {, } \\
& Q_{3}=\left\{\sigma_{3}\right\} \text {, } \\
& Q_{4}=\left\{\sigma_{5}\right\}, \\
& y\left(Q_{1}\right)=y\left(\sigma_{1}\right)=0.71 \text {, } \\
& y\left(Q_{2}\right)=y\left(\sigma_{2}\right)=0.55 \text {, } \\
& y\left(Q_{3}\right)=y\left(\sigma_{3}\right)=0.9 \text {, } \\
& y\left(Q_{4}\right)=y\left(\sigma_{5}\right)=0.8 \text {. }
\end{aligned}
$$

Once more, the pulse is deleted in PNs in the MCS whose danger degree is less than $\lambda_{y}$. Accordingly, the number vectors of PNs and their corresponding pulse values of fault pulses are updated, i.e., $\mathbf{N}_{p_{4}}^{-}=\left(1,0,1,0,1, \mathbf{O}_{21}\right)^{T}$ and $\boldsymbol{\theta}_{p_{4}}=(0.8,0,0.9,0,0.8$, $\left.\mathbf{O}_{21}\right)^{T}$, respectively.

When $k=5, \mathbf{N}_{r_{5}}^{-}=\left(\mathbf{O}_{18}\right)^{T}$.

Thus, the termination condition is satisfied and the reasoning stops. We find that the rMFRSNPS-based abductive reasoning model is shown in Figure 8, where represents the deleted pulse. Then, the fault paths can be found in Figure 8, i.e., $L_{1}=\left(\sigma_{1}, \sigma_{17}, \sigma_{30}, \sigma_{41}, \sigma_{46}\right), L_{2}=\left(\sigma_{3}, \sigma_{19}, \sigma_{30}, \sigma_{41}, \sigma_{46}\right)$, and $L_{3}=\left(\sigma_{5}, \sigma_{21}, \sigma_{32}, \sigma_{41}, \sigma_{46}\right)$, where $\sigma_{1}, \sigma_{3}$, and $\sigma_{5}$ are the fault source of "insulation winding burned down." Besides, the occurrence probability of each fault path is $P\left(L_{1}\right)=0.159, P\left(L_{2}\right)=0.217$, and $P\left(L_{3}\right)=0.186$.

Then, maintenance personnels can check the motor in turn according to the fault sources and paths got by Algorithm 3. The check order of fault sources is $\sigma_{3}, \sigma_{1}, \sigma_{5}$, and the fault paths are $L_{2}, L_{3}, L_{1}$.
4.4. Comparisons. In this section, the usefulness of the proposed method is justified by comparison with different approaches: the method of selection of amplitudes of frequencies (MSAF-12) [2], improved artificial ant clustering (IAAC) [14], fuzzy fault Petri net (FFPN) [23], and fuzzy Petri net (FPN) [44] for the abductive fault diagnosis.

Historical statistics and expertise [37] show that most faults of three-phase induction motors are related to bearings, windings, and stators. Consequently, five relevant typical cases have been considered, which are "insulation winding burned down," "excessive wear of bearing," "motor overheating," "motor overheating and abnormal rotation or the rotor is stuck," and "phase-absent operation, abnormal rotation, or the rotor is stuck and excessive wear of bearing," respectively.

The experimental results are shown in Table 2, where cases 1-3 are single faults while cases 4-5 are multiple ones. For cases 1-3, the FFPN find more fault causes and fault sources, while the FPN and IAAC diagnose more fault causes and cannot find any fault source. Besides, although the MSAF-12 can obtain right fault causes without redundant ones for case 2, it gets wrong results for cases 1 and 3. For cases 4-5, the FFPN still cannot find the accurate fault causes and fault sources, while the MSAF-12, FPN, and IAAC are unable to find any fault source. In contrast, the rMFRSNPS performs better, finding all the sources and avoiding redundancies. Accordingly, the inspection and repair scope for the motor obtained by our method is smaller than the ones got by the MSAF-12, IAAC, FFPN, and FPN.

\section{Conclusions}

This paper proposes a fault analysis method for three-phase induction motors based on rMFRSNPSs. Firstly, fault fuzzy production rules are proposed, and then, an rMFRSNPSbased fault diagnosis model is established according to them. Then, the PVRA (Algorithm 1), the FFPRA (Algorithm 2), and the BAFDRA (Algorithm 3) are designed to realize the fault analysis of motors. Specifically, the pulse value of spikes in neurons predict propagation paths with occurrence probabilities, and failure causes with probabilities are obtained by the abovementioned three algorithms in turn, respectively.

Finally, the fault diagnosis method based on rMFRSNPSs is proposed, where the FPRA can effectively predict potential failures of motors to reduce the fault rate, while the BAFDRA can carry out the abductive fault diagnosis of any failure in the proposed model to the detection range of fault sources and failures. In this paper, we extend the spectrum of applications of SNPSs to the fault analysis of motors, which not only expands the application fields of membrane computing but also extends the SNPS-based fault analysis from postante applications to a new ex-ante analysis and prediction framework. Moreover, the proposed method can meet the needs of a motor for its overall fault analysis. Case studies with a detailed reasoning process assess the feasibility and effectiveness of the proposed method. This paper focuses 
on proposing the fault analysis method and designing related algorithms from a mathematical point of view. Besides, some of our planned lines of future work include the systematic research about the software simulation of these methods, along with the in-depth exploration of practical applications where the proposed method can provide a significant value.

\section{Nomenclature}

$\begin{array}{ll}\text { ES: } & \text { Expert system } \\ \text { ANN: } & \text { Artificial neural network } \\ \text { PN: } & \text { Petri net } \\ \text { SNPS: } & \text { Spiking neural P system } \\ \text { rMFRSNPSs: } & \text { Modified fuzzy reasoning spiking neural P } \\ & \text { systems with real numbers } \\ \text { PVRA: } & \text { Pulse value reasoning algorithm } \\ \text { FFPRA: } & \text { Forward fault prediction reasoning algorithm } \\ \text { BAFDRA: } & \text { Backward abductive fault diagnosis reasoning } \\ & \text { algorithm } \\ \text { MCS: } & \text { Minimum cut set } \\ \text { PMCS: } & \text { Precise minimum cut set } \\ \text { PN: } & \text { Proposition neuron } \\ \text { RN: } & \text { Rule neuron } \\ \text { MSAF-12: } & \text { Method of selection of amplitudes of } \\ & \text { frequencies } \\ \text { FPN: } & \text { Fuzzy petri net } \\ \text { FFPN: } & \text { Fuzzy fault petri net } \\ \text { IAAC: } & \text { Improved artificial ant clustering. }\end{array}$

\section{Data Availability}

The data used to support the findings of this study are available from the corresponding author upon request.

\section{Conflicts of Interest}

All authors declare that they have no conflicts of interest.

\section{Acknowledgments}

This research was partially funded by grants from the National Natural Science Foundation of China (61703345), the Key Fund Project of the Sichuan Provincial Education Department (18ZA0459), the Chunhui Project Foundation of the Education Department of China (Z201980), the Key Scientific Research Fund Project of Xihua University (Z17108), the Open Research Subject of Key Laboratory of Fluid and Power Machinery (Xihua University), the Ministry of Education (szjj201927), and the Young Scholars Reserve Talents Support Project of Xihua University. The work of the two authors from Spain was also partially supported by the research project TIN2017-89842-P, cofinanced by Ministerio de Economía, Industria y Competitividad (MINECO) of Spain, through the Agencia Estatal de Investigación (AEI), and by Fondo Europeo de Desarrollo Regional (FEDER) of the European Union.

\section{References}

[1] S. Shi, Y. Sun, X. Li, H. Dan, and M. Su, "Moving integration filter-based open-switch fault-diagnosis method for threephase induction motor drive systems," IEEE Transactions on Transportation Electrification, vol. 6, no. 3, pp. 1093-1103, 2020.

[2] A. Glowacz, W. Glowacz, J. Kozik et al., "Detection of deterioration of three-phase induction motor using vibration signals," Measurement Science Review, vol. 19, no. 6, pp. 241-249, 2019.

[3] A. Glowacz, "Fault detection of electric impact drills and coffee grinders using acoustic signals," Sensors, vol. 19, no. 2, pp. 1-22, 2019.

[4] A. Glowacz, "Recognition of acoustic signals of commutator motors," Applied Sciences, vol. 8, no. 12, pp. 1-21, 2018.

[5] L. Maraaba, Z. Al-Hamouz, and M. Abido, "An efficient stator inter-turn fault diagnosis tool for induction motors," Energies, vol. 11, no. 3, pp. 1-18, 2018.

[6] Y. G. Xue and Y. Deng, "Refined expected value decision rules under orthopair fuzzy environment," Mathematics, vol. 8, no. 3, pp. 1-14, 2020.

[7] D. A. Ribeiro, D. D. Ferreira, D. A. Pereira, R. A. Braga Junior, and R. D. Nunes, "Mechanical fault detection in electric motors measured by a digital signal processing device in an optical mouse," Measurement, vol. 138, pp. 350-355, 2019.

[8] Y. Huang, C.-H. Chen, and C.-J. Huang, "Motor fault detection and feature extraction using RNN-based variational autoencoder," IEEE Access, vol. 7, pp. 139086-139096, 2019.

[9] W. Ahmad, S. A. Khan, and J.-M. Kim, "A hybrid prognostics technique for rolling element bearings using adaptive predictive models," IEEE Transactions on Industrial Electronics, vol. 65, no. 2, pp. 1577-1584, 2018.

[10] J. H. Lee, J. H. Pack, and I. S. Lee, "Fault diagnosis of induction motor using convolutional neural network," Applied SciencesBasel, vol. 9, no. 15, pp. 1-10, 2019.

[11] D. Y. Ning, J. Y. Hou, Y. J. Gong et al., “Auto-identification of engine fault acoustic signal through inverse trigonometric instantaneous frequency analysis," Advances in Mechanical Engineering, vol. 8, no. 3, pp. 1-8, 2016.

[12] E. Elbouchikhi, V. Choqueuse, F. Auger, and M. E. H. Benbouzid, "Motor current signal analysis based on a matched subspace detector," IEEE Transactions on Instrumentation and Measurement, vol. 66, no. 12, pp. 3260-3270, 2017.

[13] X. Yu, F. Dong, E. Ding, S. Wu, and C. Fan, "Rolling bearing fault diagnosis using modified LFDA and EMD with sensitive feature selectionfied LFDA and EMD with sensitive feature selection," IEEE Access, vol. 6, pp. 3715-3730, 2018.

[14] A. Soualhi, G. Clerc, and H. Razik, "Detection and diagnosis of faults in induction motor using an improved artificial ant clustering technique," IEEE Transactions on Industrial Electronics, vol. 60, no. 9, pp. 4053-4062, 2013.

[15] J. Burriel-Valencia, R. Puche-Panadero, J. Martinez-Roman et al., "Automatic fault diagnostic system for induction motors under transient regime optimized with expert systems," Electronics, vol. 8, no. 1, pp. 1-16, 2019.

[16] G. Rajamany, S. Srinivasan, K. Rajamany, and R. K. Natarajan, "Induction motor stator interturn short circuit fault detection in accordance with line current sequence components using artificial neural network," Journal of Electrical and Computer Engineering, vol. 2019, Article ID 4825787, 11 pages, 2019.

[17] R. F. Ribeiro Junior, F. A. de Almeida, and G. F. Gomes, "Fault classification in three-phase motors based on vibration signal 
analysis and artificial neural networks," Neural Computing and Applications, vol. 32, no. 18, pp. 15171-15189, 2020.

[18] A. Abdullah, "Ultrafast transmission line fault detection using a DWT-based ANN," IEEE Transactions on Industry Applications, vol. 54, no. 2, pp. 1182-1193, 2018.

[19] Y. T. Chen, J. C. Lai, Y. M. Jheng et al., "Partial discharge detection for stator winding insulation of motors using artificial neural network," Advances in Mechanical Engineering, vol. 10, no. 7, pp. 1-10, 2018.

[20] O. Noureldeen and I. Hamdan, "A novel controllable crowbar based on fault type protection technique for DFIG wind energy conversion system using adaptive neuro-fuzzy inference system," Protection and Control of Modern Power Systems, vol. 3, no. 1, pp. 328-339, 2018.

[21] X. Z. Cheng, C. G. Wang, J. M. Li et al., "Adaptive fault diagnosis of motors using comprehensive learning particle swarm optimizer with fuzzy petri net," Computing and Informatics, vol. 39, no. 1-2, pp. 246-263, 2020.

[22] A. T. Radhi, "Detection and localization of asymmetry in stator winding of three phase induction motors based on fuzzy neural petri net," Journal of Engineering Science and Technology, vol. 15, no. 4, pp. 2379-2394, 2020.

[23] X. Z. Cheng, C. Wang, Y. J. Yu et al., "An approach for threephase asynchronous motor failure analysis based on fuzzy fault petri net," Transactions of China Electrotechnical Society, vol. 30, no. 17, pp. 132-139, 2015.

[24] B. S. Song, X. X. Zeng, M. Jiang et al., "Monodirectional tissue P systems with promoters," IEEE Transactions on Cybernetics, vol. 51, no. 1, pp. 438-450, 2020.

[25] B. Song, X. Zeng, and A. Rodríguez-Patón, "Monodirectional tissue P systems with channel states," Information Sciences, vol. 546, pp. 206-219, 2021.

[26] L. Pan, A. Alhazov, H. Su, and B. Song, "Local synchronization on asynchronous tissue $\mathrm{P}$ systems with symport/ antiport rules," IEEE Transactions on Nanobioscience, vol. 19, no. 2, pp. 315-320, 2020.

[27] T. Wang, X. Wei, T. Huang et al., "Modeling fault propagation paths in power systems: a new framework based on event SNP systems with neurotransmitter concentration," IEEE Access, vol. 7, no. 1, pp. 12798-12808, 2019.

[28] H. N. Rong, K. Yi, G. X. Zhang, J. Dong, P. Paul, and Z. Huang, "Automatic implementation of fuzzy reasoning spiking neural $\mathrm{P}$ systems for diagnosing faults in complex power systems," Complexity, vol. 2019, Article ID 2635714, 16 pages, 2019.

[29] T. Wang, W. Liu, J. B. Zhao et al., "A rough set-based bioinspired fault diagnosis method for electrical substations," International Journal of Electrical Power \& Energy Systems, vol. 119, Article ID 105961, 2020.

[30] T. Wu, T. Zhang, and F. Xu, "Simplified and yet turing universal spiking neural $\mathrm{P}$ systems with polarizations optimized by anti-spikes," Neurocomputing, vol. 414, pp. 255-266, 2020.

[31] S. X. Jiang, J. H. Fan, Y. J. Liu et al., "Spiking neural P systems with polarizations and rules on synapses," Complexity, vol. 2020, Article ID 8742308, 12 pages, 2020.

[32] T. Wu, A. Paun, Z. Zhang, and L. Pan, "Spiking neural P systems with polarizations," IEEE Transactions on Neural Networks and Learning Systems, vol. 29, no. 8, pp. 3349-3360, 2018.

[33] T. Wang, X. G. Wei, J. Wang et al., "A weighted corrective fuzzy reasoning spiking neural $P$ system for fault diagnosis in power systems with variable topologies," Engineering
Applications of Artificial Intelligence, vol. 92, pp. 1-15, Article ID 103680, 2019.

[34] W. Liu, T. Wang, T. L. Zang et al., "A fault diagnosis method for power transmission networks based on spiking neural $\mathrm{P}$ systems with self-updating rules considering biological apoptosis mechanism," Complexity, vol. 2020, Article ID 2462647, 18 pages, 2020.

[35] Y. Deng, "Uncertainty measure in evidence theory," Science China Information Sciences, vol. 63, no. 11, pp. 1-19, 2020.

[36] Y. Xue and Y. Deng, "Entailment for intuitionistic fuzzy sets based on generalized belief structures," International Journal of Intelligent Systems, vol. 35, no. 6, pp. 963-982, 2020.

[37] R. Loiselle, Z. Xu, and I. Voloh, "Essential motor health monitoring: making informed decisions about motor maintenance before a failure occurs," IEEE Industry Applications Magazine, vol. 24, no. 6, pp. 8-13, 2018.

[38] X. Liang, M. Z. Ali, and H. Zhang, "Induction motors fault diagnosis using finite element method: a review," IEEE Transactions on Industry Applications, vol. 56, no. 2, pp. 1205-1217, 2020.

[39] P. Y. Chen, K. H. Chao, and Y. C. Tseng, "A motor fault diagnosis system based on cerebellar model articulation controller," IEEE Access, vol. 7, pp. 120326-120336, 2019.

[40] M. B. Abd-el-Malek, A. K. Abdelsalam, and O. E. Hassan, "Novel approach using Hilbert transform for multiple broken rotor bars fault location detection for three phase induction motor," ISA Transactions, vol. 80, pp. 439-457, 2018.

[41] Z. Zhao, S. Wu, B. Qiao, S. Wang, and X. Chen, "Enhanced sparse period-group lasso for bearing fault diagnosis," IEEE Transactions on Industrial Electronics, vol. 66, no. 3, pp. 2143-2153, 2019.

[42] J. Kang, L. Sun, and C. Guedes Soares, "Fault tree analysis of floating offshore wind turbines," Renewable Energy, vol. 133, pp. 1455-1467, 2019.

[43] X. Cao and Y. Deng, "A new geometric mean FMEA method based on information quality," IEEE Access, vol. 7, no. 1, pp. 95547-95554, 2019.

[44] K. Q. Zhou, W. H. Gui, L. P. Mo et al., "A bidirectional diagnosis algorithm of fuzzy petri net using inner-reasoningpath," Symmetry-Basel, vol. 10, no. 6, pp. 1-15, 2018. 